\title{
Fusion Categories Arising from Semisimple Lie Algebras
}

\author{
Henning Haahr Andersen, Jan Paradowski \\ Matematisk Institut, Aarhus Universitet, Ny Munkegade, DK-8000 Aarhus C, Denmark
}

Received: 22 November 1993

\begin{abstract}
Using tilting modules we equip certain semisimple categories with a "reduced" tensor product structure. The fusion rules for this tensor product are determined via known character formulas for the involved modules.
\end{abstract}

\section{Introduction}

In this paper the term "fusion rules" will cover the problem of describing the various decomposition multiplicities of the tensor structure on a given rigid braided tensor category.

Given a finite type Cartan datum one can associate at least 4 interesting categories to this. Namely

(1) The category (9) of the corresponding semisimple Lie algebra $\mathfrak{g}$.

(2) The category of rational modules of the corresponding semisimple, simply connected algebraic group $G$ defined over a field of positive characteristic.

(3) The category of locally finite modules of the associated quantum algebra $U$ specialized at an $l^{\text {th }}$ root of unity.

(4) The category $\tilde{\mathscr{\sigma}}_{\kappa}$ of fixed level representations of the affine Kac-Moody algebra $\tilde{\mathfrak{g}}$ associated to $\mathfrak{g}$.

In each of the cases (1-3) we shall investigate a certain semisimple subcategory equipped with a "reduced" tensor product and we shall prove some "fusion rules" in each case; these will be given in terms of the characters of the involved modules. Hence they will of course essentially be old character formulas in a new guise. Our approach will be in the framework of tilting modules.

The last case is treated in the thesis by Finkelberg [F] where he uses the "levelpreserving" tensor product on the category (ூ) (see Kazhdan and Lusztig [KL2]) to relate the Grothendieck ring of $\tilde{\mathscr{O}}_{\kappa}$ with a quotient of $\mathscr{R}$ defined in 1.15 . We state his precise result in 1.24 .

The paper is divided into 3 parts dealing with the three above mentioned situations (1-3) respectively. Also in the first part we investigate the category $\mathscr{Q}_{S}$ and in the 
third part we show how to avoid any restrictions on $l$. For the latter we need a modification due to Lusztig of the quantum Frobenius homomorphism (also due to Lusztig).

\section{Notation}

Throughout this paper the following notations will be used:

- $I$ a finite set.

- $\left(a_{i j}\right)_{\imath, \jmath \in I}$ a (finite type) Cartan matrix and $d_{\imath} \in\{1,2,3\}$ chosen minimal such that $\left(d_{i} a_{i j}\right)$ is symmetric.

- $\mathfrak{g}$ the semisimple Lie algebra corresponding to $\left(a_{i j}\right)$.

- $\mathfrak{h}$ a Cartan subalgebra of $\mathfrak{g}$.

- $\mathfrak{b}$ a Borel subalgebra of $\mathfrak{g}$ containing $\mathfrak{h}$.

- $R \subset \mathfrak{h}^{*}$ the corresponding root system and $R^{+}$the set of positive roots w.r.t. $\mathfrak{b}$.

- $G$ a semisimple, simply connected algebraic group defined over an algebraically closed field $\bar{k}$ of characteristic $p>0$.

- $h$ is the Coxeter number for the root system.

- $B$ a Borel subgroup of $G$ containing a maximal torus $T$ such that the roots of $B$ are the negative ones.

- $W$ the Weyl group associated to all these data. The longest element in $W$ is called $w_{0}$.

\section{The Categories $\mathscr{Q}$ and $\mathscr{Q}_{S}$}

1.1. Fix $S \subseteq I$. We shall identify elements in $S$ with their corresponding simple roots. Let $R_{S}$ and $W_{S}$ denote the root system and the Weyl group associated to $S$. The integral weights are $X=\left\{\zeta \in \mathfrak{h}^{*} \mid\langle\zeta, \check{\alpha}\rangle \in \mathbb{Z}\right\}, X^{+}$denotes the set of dominant integral weights and we let $\varrho$ be half the sum of the positive roots. Let $W$ act on $\mathfrak{h}^{*}$ by $w \cdot \lambda=w(\lambda+\varrho)-\varrho$. A weight $\lambda \in X$ will be called $S$-dominant and we write $\lambda \in X_{S}^{+}$if $\langle\lambda, \check{\alpha}\rangle \geq 0$ for all $\alpha \in S$.

Let $\mathscr{O}$ denote the category consisting of all finitely generated $\mathfrak{g}$-modules which are $\mathfrak{h}$-semisimple and $\mathfrak{b}$-finite. If $M \in \mathscr{Q}$ and $\lambda \in \mathfrak{h}^{*}$ the $\lambda$-weight space of $M$ is

$$
M_{\lambda}=\{m \in M \mid h . m=\lambda(h) m, h \in \mathfrak{h}\}
$$

and $\lambda$ is called a weight of $M$ if $M_{\lambda} \neq 0$. We assume that all weights of the modules we consider are integral.

The category $\mathscr{Q}_{S}$ is the full subcategory of $\mathscr{Q}$ consisting of those $M \in \mathscr{O}$ for which all composition factors have highest weights in $X_{S}^{+}$. We see that the choice $S=\emptyset$ gives us the $\odot$ setup back and therefore we will drop the subscript $\emptyset$ everywhere. Note however that $X_{\emptyset}^{+}=X$ and not $X^{+}$.

Among the modules in $(2)$ we have the Verma modules $M(\lambda), \lambda \in X$. Each Verma module $M(\lambda)$ has a unique simple quotient $L(\lambda)$ and these are up to isomorphism all the simple modules in $\mathscr{O}$. All modules in $\mathcal{O}$ have finite lengths.

Clearly the simple modules in $\mathscr{Q}_{S}$ are $\left\{L(\lambda) \mid \lambda \in X_{S}^{+}\right\}$. On the other hand the Verma modules does not belong to $\mathscr{\mathscr { C }}_{S}$ (not even those with highest weights in $X_{S}^{+}$). However the generalized Verma modules defined as follows will: Denote by $\mathfrak{p}_{S} \subset \mathfrak{g}$ the parabolic subalgebra corresponding to $S$. For $\lambda \in X_{S}^{+}$we denote by $L_{S}(\lambda)$ the 
finite dimensional simple $\mathfrak{p}_{S}$-module with highest weight $\lambda$. Then the generalized Verma module is

$$
M_{S}(\lambda):=U(\mathfrak{g}) \otimes_{U\left(\mathfrak{p}_{S}\right)} L_{S}(\lambda) .
$$

Clearly $M_{S}(\lambda)$ has highest weight $\lambda$ and $L(\lambda)$ is its unique simple quotient.

1.2. We denote by $\mathscr{K}$ the Grothendieck group of $\mathscr{Q}$. Clearly this is the free Abelian group on $\{[L(\lambda)] \mid \lambda \in X\}$ when we denote the image of $M \in \mathscr{O}$ in $\mathscr{K}$ by $[M]$. Similarly the Grothendieck group $\mathscr{K}_{S}$ of $\mathscr{Q}_{S}$ is the free Abelian group with basis $\left\{[L(\lambda)] \mid \lambda \in X_{S}^{+}\right\}$. We have

$$
[M]=\sum_{\lambda \in X}[M: L(\lambda)][L(\lambda)]
$$

where $[M: L(\lambda)]$ denotes the composition factor multiplicity of $L(\lambda)$ in $M$. In particular we have

$$
[M(\lambda)]=\sum_{\mu \leq \lambda}[M(\lambda): L(\mu)][L(\mu)]
$$

which has only finitely many non-zero terms. Using that for all $\lambda \in X$,

$$
[L(\lambda): M(\lambda)]=[M(\lambda): L(\lambda)]=1 .
$$

we can invert $(*)$ and get

$$
[L(\lambda)]=\sum_{\mu \leq \lambda}[L(\lambda): M(\mu)][M(\mu)]
$$

where $[L(\lambda): M(\mu)] \in \mathbb{Z}$. In general the numbers $[L(\lambda): M(\mu)]$ and $[M(\lambda): L(\mu)]$ are determined by the Kazhdan-Lusztig conjecture [KL1] (which has been a theorem for 13 years, see $[\mathrm{BB}]$ and $[\mathrm{BK}])$.

We also note that $\left\{\left[M_{S}(\lambda)\right] \mid \lambda \in X_{S}^{+}\right\}$is a basis for $\mathscr{K}_{S}$. If we consider $\mathscr{K}_{S}$ as a subgroup of $\mathscr{K}$ we have the character formula

$$
\left[M_{S}(\lambda)\right]=\sum_{w \in W_{S}}(-1)^{l(w)}[M(w \cdot \lambda)], \quad \lambda \in X_{S}^{+}
$$

This is obtained from the identity $\left[L_{S}(\lambda)\right]=\sum_{\lambda \in W_{S}}(-1)^{l(w)}\left[U\left(p_{S}\right) \otimes_{U(\mathfrak{b})} w \cdot \lambda\right]$ by induction to $\mathfrak{g}$. This identity on the other hand can be found in $[\mathrm{H}, 24.2]$.

Recall that $\mathscr{Q}_{S}$ decomposes as

$$
\mathscr{Q}_{S}=\bigoplus_{\substack{\lambda \in X_{S}^{+} \\ \lambda+\varrho \in X^{+}}} \mathscr{\sigma}_{S}(\lambda)
$$

where $\mathscr{C}_{S}(\lambda)$ is the full subcategory of $\overbrace{S}$ consisting of those $M \in \overbrace{S}$ for which $[M: L(\nu)]$ is only non-zero when $\nu \in W \cdot \lambda$.

If $M \in \mathscr{O}$ we write $p_{\lambda}(M) \in \mathscr{O}(\lambda)$ for the largest submodules of $M$ in $\mathscr{Q}(\lambda)$. Furthermore recall that if $\lambda \in X^{+}$then $L(\lambda)$ is finite dimensional and clearly $M \otimes L(\lambda) \in \mathscr{O}$ for all $\lambda \in X^{+}$. For $\lambda+\varrho, \mu+\varrho \in X^{+}$we have the translation functor

$$
T_{\lambda}^{\mu}: \mathscr{O}(\lambda) \rightarrow \mathscr{Q}(\mu)
$$


given by $T_{\lambda}^{\mu}(M)=p_{\mu}(M \otimes L(w(\mu-\lambda)))$, where $w \in W$ is chosen such that $w(\mu-\lambda) \in X^{+}$. Then $T_{\lambda}^{\mu}$ and $T_{\mu}^{\lambda}$ are adjoint functors.

If $\lambda$ and $\mu$ as above happen to be in $X_{S}^{+}$then the translation functors restrict to adjoint functors (denoted the same way)

$$
T_{\lambda}^{\mu}: \mathscr{\odot}_{S}(\lambda) \rightarrow \mathscr{Q}_{S}(\mu) \text { and } T_{\mu}^{\lambda}: \mathscr{Q}_{S}(\mu) \rightarrow \mathscr{\odot}_{S}(\lambda) \text {. }
$$

1.3. The behaviour of Verma modules under translation is easy to describe. First one observes that for $\lambda \in X$ and $\mu \in X^{+}$the module $M(\lambda) \otimes L(\mu)$ has a filtration by Verma modules (a so-called Verma flag):

$$
0=F_{0} \subset F_{1} \subset \ldots \subset F_{r}=M(\lambda) \otimes L(\mu)
$$

with $F_{i} / F_{i-1} \cong M\left(\lambda+\nu_{\imath}\right), i=1, \ldots, r$ and $\nu_{i}$ running through the weights of $L(\mu)$. In the Grothendieck group $\mathscr{T}$ this means that we have (with obvious notation):

$$
[M(\lambda) \otimes L(\mu): M(\lambda+\nu)]=\operatorname{dim} L(\mu)_{\nu}
$$

for all $\nu$. Simple weight considerations then give that whenever $\lambda \in X^{+}$,

$$
T_{\lambda}^{\mu} M(w \cdot \lambda)=M(w \cdot \mu) \text { for all } \mu+\varrho \in X^{+}, w \in W
$$

and $T_{\mu}^{\lambda} M(w \cdot \mu)$ has a Verma flag with

$$
\left[T_{\mu}^{\lambda} M(w \cdot \mu): M(\nu)\right]= \begin{cases}1 & \text { for } \nu \in W_{\mu} \cdot \lambda \\ 0 & \text { otherwise } .\end{cases}
$$

Here $W_{\mu}$ is the stabilizer in $W$ of $\mu$ under the dot-action.

We are also interested in the effect of the translation functors on generalized Verma modules. So suppose that $\lambda \in X^{+}$and let $w \in W$ such that $w \cdot \lambda \in X_{S}^{+}$. Then

$$
T_{\lambda}^{\mu} M_{S}(w \cdot \lambda)= \begin{cases}M_{S}(w \cdot \mu) & \text { if } w \cdot \mu \in X_{S}^{+} \\ 0 & \text { otherwise }\end{cases}
$$

Suppose in addition that $W_{\mu}=\{1, s\}$ for some simple reflection $s$. For all $w \in W$ with $w . \mu \in X_{S}^{+}$we have an exact sequence

$$
0 \rightarrow M_{S}(w . \lambda) \rightarrow T_{\mu}^{\lambda} M_{S}(w . \mu) \rightarrow M_{S}(w s . \lambda) \rightarrow 0,
$$

if $w . \lambda>w s . \lambda$, resp.

$$
0 \rightarrow M_{S}(w s \cdot \lambda) \rightarrow T_{\mu}^{\lambda} M_{S}(w \cdot \mu) \rightarrow M_{S}(w \cdot \lambda) \rightarrow 0,
$$

if $w . \lambda<w s . \lambda$.

1.4. The effect of the translation functors on simple modules is more complicated to describe. We shall only need the following case. Suppose $\lambda+\varrho, \mu+\varrho \in X^{+}$with $W_{\lambda}=1$. Then

$$
T_{\lambda}^{\mu} L(w \cdot \lambda)= \begin{cases}L(w \cdot \mu) & \text { if } w s . \lambda>w \cdot \lambda \text { for all } s \in W_{\mu} \\ 0 & \text { otherwise }\end{cases}
$$

For this as well as the results in 1.3 see [J1].

1.5. On the category $\mathscr{O}$ we have the "contravariant dual" functor $\delta$. It is given by the following recipe. Let $M \in \mathscr{O}$. Then $\delta M=\bigoplus_{\lambda \in X} \operatorname{Hom}_{\mathbb{C}}\left(M_{\lambda}, \mathbb{C}\right)$ as a vector space. 
Then $\delta M \subseteq M^{*}$ and the $\mathfrak{g}$-module structure on $\delta M$ is inherited from the action of $\mathfrak{g}$ on $M^{*}$ given as

$$
x \cdot f: m \mapsto f(\tau(x) m), \quad \text { for } \quad x \in \mathfrak{g}, f \in M^{*}, m \in M,
$$

where $\tau$ is the antiautomorphism of $\mathfrak{g}$ which fixes $\mathfrak{h}$ and takes the Chevalley basis element $e_{\alpha}$ into $e_{-\alpha}, \alpha \in R^{+}$. Clearly $\delta^{2}=1$ and noting that the weights of $\delta M$ are the same as the weights of $M$ it follows that for $\lambda \in X$,

$$
\delta L(\lambda) \cong L(\lambda) .
$$

This functor obviously also restricts to $\sigma_{S}$.

Following Donkin [D] we make the

Definition. A module $M \in \mathcal{Q}^{\prime}$ is called a (partial) tilting module if $M$ and $\delta M$ both have Verma flags.

A module $M \in C_{S}$ is called a tilting module if $M$ and $\delta M$ both have generalized Verma flags (i.e. filtrations in which successive quotients are generalized Verma modules).

Clearly it amounts to the same to say that $M$ is a tilting module if and only if $M$ both has a (generalized) Verma flag and a dual (generalized) Verma flag (i.e. a filtration with subquotients of the form $\delta M(\lambda)$ for some $\lambda \in X$.)

Note that

(2) Direct sums, tensor products with finite dimensional modules, and direct summands of tilting modules are tilting modules.

(3) Translation functors send tilting modules to tilting modules. (This follows from 1.3.)

(4) $M(\lambda)$ is a tilting module iff $M(\lambda)=L(\lambda)$ iff $w_{0}(\lambda)+\varrho \in X^{+}$. (This follows directly from the definition and (1).)

(5) $M_{S}(\lambda)$ is a tilting module iff $M_{S}(\lambda)=L(\lambda)$ iff $\langle\lambda+\varrho, \check{\alpha}\rangle<0$ for all $\alpha \in R^{+} \backslash R_{S}^{+}$.

1.6. We record the following well-known

Lemma. For all $\lambda, \mu \in X$ we have $\operatorname{Ext}_{C}^{1}(M(\lambda), \delta M(\mu))=0$.

Proof. Suppose $0 \rightarrow \delta M(\mu) \rightarrow E \rightarrow M(\lambda) \rightarrow 0$ is an exact sequence in $\mathscr{Q}$. If $\mu \ngtr \lambda$ then $\lambda$ is a maximal weight in $E$ and by the universal property of $M(\lambda)$ this means that there is a section $M(\lambda) \rightarrow E$. On the other hand if $\mu>\lambda$ we apply $\delta$ to our exact sequence and obtain the exact sequence $0 \rightarrow \delta M(\lambda) \rightarrow \delta E \rightarrow M(\mu) \rightarrow 0$. But this splits by the argument we just used and hence so does the original sequence.

Remark. In fact $\operatorname{Ext}_{C}^{2}(M, N)=0$ for $i>0$ whenever $M$ has a Verma flag and $N$ has a dual Verma flag. Adding the subscript $S$ all over this carries over to $\mathscr{C}_{S}$.

1.7. We have the following version of the general results of Ringel [R] and Donkin [D] on tilting modules.

Proposition. Let $\lambda \in X$. Then there exists an indecomposable tilting module $D(\lambda) \in$ (2) such that

(1) every weight $\mu$ of $D(\lambda)$ satisfy $\mu \leq \lambda$.

(2) $\operatorname{dim} D(\lambda)_{\lambda}=1$.

Moreover $D(\lambda)$ is unique up to isomorphism. 
If $\lambda \in X_{S}^{+}$then there exists a unique (up to isomorphism) indecomposable tilting module $D_{S}(\lambda) \in \mathscr{G}_{S}$ such that

(3) every weight $\mu$ of $D_{S}(\lambda)$ satisfy $\mu \leq \lambda$.

(4) $\operatorname{dim} D_{S}(\lambda)_{\lambda}=1$.

Proof $[\mathrm{CI}]$. We give the proof in the category $\mathscr{C}$-case. (Of course the proposition is a special case of the general result on the existence of tilting modules [R].) Suppose $\lambda+\varrho$ is dominant. Then $D\left(w_{0} \cdot \lambda\right)=M\left(w_{0} \cdot \lambda\right)$ has the required properties, see 1.5(4). We shall proceed by descending induction on $w \in W$ to show that $D(w \cdot \lambda)$ exists. So suppose $w \neq w_{0}$ and pick a simple reflection $s$ such that $w s>w$ (in the Chevalley order). If $s$ may be chosen such that $w s . \lambda=w \cdot \lambda$ there is nothing to prove. Otherwise we choose $\mu$ with $\mu+\varrho \in X^{+}$and $s \in W_{\mu}$. Then $D(w . \mu)$ exists by the induction hypothesis. Consider $T_{\mu}^{\lambda} D(w . \mu)$. This is a tilting module according to 1.5(3). Looking at the Verma flag for $T_{\mu}^{\lambda} D(w . \mu)$ we see (in view of the results in 1.3) that $w . \lambda$ is a maximal weight in this module and that it occurs with multiplicity 1 . It follows that there is a unique indecomposable summand $D(w \cdot \lambda)$ of $T_{\mu}^{\lambda} D(w \cdot \mu)$ such that $D(w \cdot \lambda)_{w . \lambda} \neq 0$. Clearly $D(w \cdot \lambda)$ has the required properties.

To prove uniqueness suppose, for a given $\lambda \in X$, that both $D(\lambda)$ and $D^{\prime}(\lambda)$ are indecomposable tilting modules in $\odot$ with properties (1) and (2). Then we have inclusions $M(\lambda) \subset D(\lambda)$ and $M(\lambda) \subset D^{\prime}(\lambda)$ coming from the Verma flags for $D(\lambda)$ and $D^{\prime}(\lambda)$. Since $D(\lambda) / M(\lambda)$ has a Verma flag and $D^{\prime}(\lambda)$ has a dual Verma flag we see by Lemma 1.6 that $\operatorname{Ext}^{1}\left(D(\lambda) / M(\lambda), D^{\prime}(\lambda)\right)=0$. It follows that there is a homomorphism $\phi: D(\lambda) \rightarrow D^{\prime}(\lambda)$ which restricts to an inclusion on $M(\lambda)$. Likewise we obtain a homomorphism $\phi^{\prime}: D(\lambda) \rightarrow D^{\prime}(\lambda)$ with the similar property. The composite $\phi^{\prime} \circ \phi$ is then an endomorphism of $D(\lambda)$ which is an isomorphism on $D(\lambda)_{\lambda}$. Since $D(\lambda)$ is indecomposable of finite length we conclude that $\phi^{\prime} \circ \phi$ is an isomorphism. The same argument applies to $\phi \circ \phi^{\prime}$ and we are done.

1.8 Proposition [CI]. If $\lambda+\varrho \in X^{+}$then $D(\lambda)=T_{-\varrho}^{\lambda} M(-\varrho)$ and this is the projective cover in $\odot$ of $L\left(w_{0} . \lambda\right)$.

Proof. Since $M(-\varrho)$ is projective and simple in $\mathscr{O}$ and since translation clearly preserves projectivity we see from $1.5(4)$ that $T_{-\varrho}^{\lambda} M(-\varrho)$ is a projective tilting module. It has highest weight $\lambda$. Moreover using 1.4 (with $\mu=-\varrho$ ) we see via the adjointness properties of $T_{-\varrho}^{\lambda}$ and $T_{\lambda}^{-\varrho}$ that $T_{-\varrho}^{\lambda} M(-\varrho)$ has simple socle (and head) equal to $L\left(w_{0} \cdot \lambda\right)$. It is therefore indecomposable and we are done.

1.9. Proposition 1.8 does not carry over unaltered to $\mathscr{Q}_{S}$. One needs the notion of socular elements in $W_{S} \backslash W$. Fix an anti-dominant weight $\lambda \in X^{-}$. Then $w \in W_{S} \backslash W$ is called socular if $L\left(w_{S} w \cdot \lambda\right)$ appears as a summand in the socle of a $M_{S}\left(w_{S} z . \lambda\right)$ for some $z \in W_{S} \backslash W$. Here $w_{S}$ is the longest element in $W_{S}$. Note that this definition depends on $\lambda$. We refer to [I] for the proof of the following

Proposition. The projective tilting modules in $\mathscr{Q}_{S}$ are precisely those corresponding to socular elements, or equivalently

$$
P_{S}\left(w_{S} w \cdot \lambda\right) \text { is self-dual iff } w \text { is socular. }
$$

in the notation of [I].

1.10. If we combine Proposition 1.8 with $1.3(2)$ we get 
Corollary. If $\lambda \in X^{+}$then

$$
[D(\lambda): M(\mu)]= \begin{cases}1 & \text { if } \mu \in W . \lambda \\ 0 & \text { otherwise }\end{cases}
$$

1.11. From Proposition 1.8 we see that $\left\{\left[D_{S}(\lambda)\right] \mid \lambda \in X_{S}^{+}\right\}$is a basis for $\mathscr{K}_{S}$ and we have the decomposition

$$
\mathscr{K}_{S}=\mathscr{K}_{S}^{-} \oplus \mathscr{K}_{S}^{\prime},
$$

where

$$
\begin{aligned}
\mathscr{K}_{S}^{-} & =\operatorname{span}_{\mathbb{Z}}\left\{\left[D_{S}(\lambda)\right] \mid \lambda \in X_{S}^{+} \text {and }\langle\lambda+\varrho, \check{\alpha}\rangle<0 \text { for all } \alpha \in R^{+} \backslash R_{S}^{+}\right\}, \\
\mathscr{K}_{S}^{\prime} & =\operatorname{span}_{\mathbb{Z}}\left\{\left[D_{S}(\lambda)\right] \mid \lambda \in X_{S}^{+} \text {and }\langle\lambda+\varrho, \check{\alpha}\rangle \geq 0 \text { for some } \alpha \in R^{+} \backslash R_{S}^{+}\right\} .
\end{aligned}
$$

Note that in the category (9) case we can rewrite this as

$$
\begin{aligned}
\mathscr{K}^{-} & =\operatorname{span}_{\mathbb{Z}}\left\{[D(\lambda)] \mid w_{0} \cdot \lambda \in X^{+}\right\}, \\
\mathscr{K}^{\prime} & =\operatorname{span}_{\mathbb{Z}}\left\{[D(\lambda)] \mid w_{0} \cdot \lambda \notin X^{+}\right\} .
\end{aligned}
$$

Note also that by $1.5(4-5)$ the basis elements of $\mathscr{K}_{S}^{-}$are equal to $\left[M_{S}(\lambda)\right]$.

In order to obtain another characterization of $\mathscr{K}^{\prime}$ we need the following analogue of $[\mathrm{A} 1]$

Proposition. Suppose $\lambda \in X^{+}$and $\mu+\varrho \in X^{+}$with $W_{\mu}=\{1, s\}$. Then we have, for all $w \in W$ for which $w<w_{S}$,

$$
T_{\mu}^{\lambda} D(w \cdot \mu)=D(w \cdot \lambda) .
$$

Proof. It is clear that $T_{\mu}^{\lambda} D(w \cdot \mu)=D(w \cdot \lambda) \oplus D$ for some tilting module $D$. We shall prove that $D=0$ by showing that $T_{\lambda}^{\mu} D=0$. Looking at the Verma flag for $D(w \cdot \lambda)$ we observe that $w \cdot \mu$ is a highest weight of $T_{\lambda}^{\mu} D(w \cdot \lambda)$. Also $T_{\lambda}^{\mu} T_{\mu}^{\lambda} D(w \cdot \mu)=D(w \cdot \lambda) \oplus D(w \cdot \lambda)$. We are therefore done if we prove that $w \cdot \mu$ occurs with multiplicity two as a weight of $T_{\lambda}^{\mu} D(w \cdot \lambda)$. For this we consider the non-split extension from 1.3(4),

$$
0 \rightarrow M(w . \lambda) \rightarrow T_{\mu}^{\lambda} M(w \cdot \mu) \rightarrow M(w s . \lambda) \rightarrow 0 .
$$

We claim that the inclusion $M(w \cdot \lambda) \subset D(w \cdot \lambda)$ extends to an inclusion $T_{\mu}^{\lambda} M(w \cdot \mu) \subset$ $D(w . \lambda)$ (this will do it, because then $M(w \cdot \mu)=T_{\lambda}^{\mu} M(w \cdot \mu)=T_{\lambda}^{\mu} M(w s . \lambda)$ will occur twice in $T_{\lambda}^{\mu} D(w . \lambda)$.) The vanishing of $\operatorname{Ext}^{1}(M(w s . \lambda), D(w . \lambda))$ - Lemma 1.6 - shows that the inclusion $M(w \cdot \lambda) \subset D(w \cdot \lambda)$ does indeed extend to a homomorphism $\phi: T_{\mu}^{\lambda} M(w . \mu) \rightarrow D(w . \lambda)$. Since (1) is non-split we see that $\operatorname{ker} \phi$ does not have $w s . \lambda$ as a weight (if it did we would get a section $M(w s . \lambda) \rightarrow \operatorname{ker} \phi \subset T_{\mu}^{\lambda} M(w . \mu)$ ). Hence we have a non-zero homomorphism $M(w s . \lambda) \rightarrow D(w \cdot \lambda) / M(w . \lambda)$. This must then be the lowest part of a Verma flag for $D(w . \lambda) / M(w . \lambda)$ and we are done.

1.12. Corollary. Let $\lambda \in X^{+}$and $w \in W$. Then $[D(w \cdot \lambda): M(y s . \lambda)]=[D(w \cdot \lambda)$ : $M(y . \lambda)]$ for all $y \in W$ and all simple reflections $s$ with $w s . \lambda<w . \lambda$.

Proof. For a given simple reflection $s$ choose $\mu+\varrho \in X^{+}$such that $W_{\mu}=\{1, s\}$. Then we have for all $y \in W$,

$$
\begin{aligned}
{[D(w \cdot \lambda): M(y \cdot \lambda)] } & =\left[T_{\mu}^{\lambda} D(w \cdot \mu): M(y \cdot \lambda)\right]=[D(w \cdot \mu): M(y \cdot \mu)] \\
& =[D(w \cdot \mu): M(y s \cdot \mu)]=\left[T_{\mu}^{\lambda} D(w \cdot \mu): M(y s \cdot \lambda)\right] \\
& =[D(w \cdot \lambda): M(y s \cdot \lambda)] .
\end{aligned}
$$


1.13. Of course both the proposition and its corollary have direct analogues in $\mathscr{Q}_{S}$. Let us state the result corresponding to the corollary.

Corollary. Let $\lambda \in X^{+}$and suppose $s$ is a simple reflection. For all $y, w \in W$ such that $y . \lambda, w . \lambda$, ws. $\lambda \in X_{S}^{+}$with $w s . \lambda<w . \lambda$ we have

$$
\left[D_{S}(w \cdot \lambda): M_{S}(y \cdot \lambda)\right]= \begin{cases}{\left[D_{S}(w \cdot \lambda): M_{S}(y s . \lambda)\right]} & \text { if ys. } \lambda \in X_{S}^{+} \\ 0 & \text { otherwise } .\end{cases}
$$

1.14. The action of $W$ on $X$ induces a $\mathbb{Z}$-linear action on $\mathscr{K}$ by

$$
w \cdot[M(\lambda)]=[M(w \cdot \lambda)], \quad \text { for } \quad \lambda \in X, w \in W .
$$

(Recall that $\{[M(\lambda)] \mid \lambda \in X\}$ is a basis for $\mathscr{K}$.) We can now characterize the summand $\mathscr{K}^{\prime}$ of $\mathscr{K}$.

Proposition. $\mathscr{K}^{\prime}=\operatorname{span}_{\mathbb{Z}}\left\{f \in \mathscr{K} \mid s_{\alpha} \cdot f=\right.$ f for some $\left.\alpha \in R\right\}$.

Proof. Let $\lambda \in X$ and suppose $w_{0} . \lambda \notin X^{+}$. If $W_{\lambda} \neq 1$ then

$$
[D(\lambda)]=\sum_{\mu: W_{\mu} \neq 1}[D(\lambda): M(\mu)][M(\mu)]
$$

Hence the Proposition is true for such $\lambda$ 's.

So assume $W_{\lambda}=1$. Then Corollary 1.12 shows that if $s$ is a reflection in one of the walls of the chamber containing $\lambda$ and $s . \lambda<\lambda$, then

$$
[D(\lambda)]=\sum_{y: y . \lambda<y s . \lambda}[D(\lambda): M(y \cdot \lambda)]([M(y \cdot \lambda)]+[M(y s \cdot \lambda)]) .
$$

Noting that

$$
y s y^{-1} \cdot([M(y \cdot \lambda)]+[M(y s \cdot \lambda)])=[M(y s \cdot \lambda)]+[M(y \cdot \lambda)],
$$

we are done.

1.15. Let $\mathscr{C}$ denote the category consisting of all finite dimensional representations of $\mathfrak{g}$. The Grothendieck group of $\mathscr{C}$ is then the free Abelian group on $\left\{[L(\lambda)] \mid \lambda \in X^{+}\right\}$. The tensor product on $\mathscr{C}$ makes this into a commutative ring with unit which we shall denote by $\mathscr{B}$. Recall that

$$
\mathscr{R} \stackrel{\sim}{\rightarrow}[X]^{W}
$$

where $\mathbb{Z}[X]$ denotes the group ring of $X$. As usual we write $e^{\lambda}$ for the basis element in $\mathbb{Z}[X]$ corresponding to $\lambda \in X$. Then $W$ acts linearly on $\mathbb{Z}[X]$ via $w e^{\lambda}=e^{w(\lambda)}$, $w \in W, \lambda \in X$ and $\mathbb{Z}[X]^{W}$ is the ring of invariants for this action. The isomorphism in (1) is given by

$$
[L(\lambda)] \mapsto \operatorname{ch} L(\lambda)=\sum_{\nu} \operatorname{dim} L(\lambda)_{\nu} e^{\nu}, \quad \lambda \in X^{+} .
$$

Recall the classical formula for the character of tensor products

$$
\operatorname{ch}(M \otimes L(\lambda))=\sum_{\nu \in X^{+}} \sum_{w \in W}(-1)^{l(w)} \operatorname{dim} M_{w \cdot \nu-\lambda} \operatorname{ch} L(\nu) .
$$


Since both sides are additive in $M$ it is enough to verify it for $M=L(\mu)$ with $\mu \in X^{+}$and in this case we refer to [A2, Final remark]. If we write in $\mathscr{B}$

$$
[L(\mu)] \cdot[L(\lambda)]=\sum_{\nu \in X^{+}} a_{\mu, \lambda}^{\nu}[L(\nu)], \quad \mu, \lambda \in X^{+},
$$

then (2) says that the structure constants $a_{\mu, \lambda}^{\nu}$ are given by

$$
a_{\mu, \lambda}^{\nu}=\sum_{w \in W}(-1)^{l(w)} \operatorname{dim} L(\mu)_{w . \nu-\lambda} .
$$

1.16. As observed before the category $\mathscr{Q}$ is stable under tensoring with a finite dimensional module. This gives the Grothendieck group $\mathscr{K}$ the structure of a module over $\mathscr{R}$, namely the structure induced by $[L] \cdot[M]=[L \otimes M], L \in \mathscr{C}, M \in \mathscr{Q}$. Note that in this language 1.3(1) says

$$
[L(\mu)] \cdot[M(\lambda)]=\sum_{\nu \in X} \operatorname{dim} L(\mu)_{\nu}[M(\lambda+\nu)], \quad \lambda \in X, \mu \in X^{+} .
$$

Lemma. The action of $W$ on $\mathscr{K}$ (see 1.14) respects the $\mathscr{B}$-module structure.

Proof. By (1) we have for all $w \in W, \mu \in X^{+}, \lambda \in X$,

$$
\begin{aligned}
w \cdot([L(\mu)] \cdot[M(\lambda)]) & =\sum_{\nu \in X} \operatorname{dim} L(\mu)_{\nu}[M(w \cdot(\lambda+\nu))] \\
& =\sum_{\nu \in X} \operatorname{dim} L(\mu)_{\nu}[M(w \cdot \lambda+w(\nu))] \\
& =\sum_{\nu \in X} \operatorname{dim} L(\mu)_{\nu}[M(w \cdot \lambda+\nu)] \\
& =[L(\mu)](w \cdot[M(\lambda)]),
\end{aligned}
$$

where we have used that $\operatorname{dim} L(\mu)_{\nu}=\operatorname{dim} L(\mu)_{w(\nu)}$.

1.17 Proposition. The additive subgroup $\mathscr{K}^{\prime}$ of $\mathscr{K}$ is an $\mathscr{B}$-submodule.

Proof. Using Lemma 1.16 this is clear from the characterization of $\mathscr{K}^{\prime \prime}$ in Proposition 1.14 .

This proposition gives us an $\mathscr{B}$-module structure on $\mathscr{K}^{-} \cong \mathscr{K} / \mathscr{K}^{\prime}$.

1.18 Proposition. As $\mathscr{R}$-modules we have $\mathscr{K}^{-} \cong \mathscr{R}$.

Proof. We claim that the additive isomorphism $\Phi: \mathscr{K}^{-} \rightarrow \mathscr{B}$ given by $\Phi\left[M\left(w_{0} \cdot \lambda\right)\right]=$ $[L(\lambda)], \lambda \in X^{+}$respects the $\mathscr{B}$-module structures. To see this we first note that since $[M(\mu)]+\left[M\left(s_{\alpha} \cdot \mu\right)\right] \in \mathscr{K}^{\prime}$ for all $\mu \in X, \alpha \in R$ we have

$$
[M(\mu)] \equiv(-1)^{l(w)}[M(w \cdot \mu)] \bmod \mathscr{K}^{\prime} \quad \text { for all } \nu \in X, w \in W .
$$

Using this we get via $1.16(1)$ the following equation in $\mathscr{K}^{-}$

$$
[L(\mu)] \cdot\left[M\left(w_{0} \cdot \lambda\right)\right]=\sum_{\nu \in X^{+}} \sum_{w \in W}(-1)^{l(w)} \operatorname{dim} L(\mu)_{w \cdot \lambda-\nu}\left[M\left(w_{0} \cdot \nu\right)\right] .
$$

Comparing with $1.15(3)$ we conclude that $\Phi$ is indeed an $\mathscr{R}$-module homomorphism. 
One could also have obtained this using results from [I]. Note that there is an $\mathscr{B}$-linear endomorphism $\Psi$ of $\mathscr{K}$ taking $[M(\lambda)]$ to $\left[M\left(w_{0} . \lambda\right)\right]$ for all $\lambda \in X$. Then in fact $\Psi([P(\lambda)])=\left[D\left(w_{0} . \lambda\right)\right]$ for all $\lambda \in X$; to see this combine Proposition 5.2.6 and Theorem 3.4.1 from [I]. Now it is easy to deduce the proposition above.

1.19. Consider the full subcategory $\mathscr{O}^{-}$of $\mathscr{O}$ consisting of all tilting modules whose composition factors have antidominant highest weights, i.e. a module $M$ belongs to $Q^{-}$if and only if

$$
M=\bigoplus_{\lambda \in X^{+}} M\left(w_{0} \cdot \lambda\right)^{a_{\lambda}(M)}
$$

for some $a_{\lambda}(M) \in \mathbb{N}$ (zero for all but finitely many $\lambda \in X^{+}$). If $N \in \mathscr{C}$ we define the reduced tensor product

$$
N \underline{\otimes} M=\bigoplus_{\lambda \in X^{+}} D(\lambda)^{a_{\lambda}(N \otimes M)} .
$$

Note that the Grothendieck group of $\mathscr{G}^{-}$is $\mathscr{K}^{-}$and the above $\mathscr{B}$-structure on $\mathscr{K}^{-}$ is given by

$$
[N] \cdot[M]=[N \underline{\otimes} M] \text { for } N \in \mathscr{C}, M \in \mathscr{O}^{-} .
$$

The proposition above (cf. the formula (1)) says that the reduced tensor product of a "dominant simple" with an "antidominant Verma" decomposes into antidominant Vermas in exactly the same manner as the usual tensor product of the corresponding two finite dimensional simple modules decomposes into simples. That such a result should hold was suggested to us by Humphreys.

These "fusion rules" give $0^{-}$the structure of a module for the rigid symmetric monoidal category $\mathscr{C}$.

1.20. We now want to prove a similar result for the category $\mathscr{\sigma}_{S}$. We cannot proceed by just "adding the subscript $S$." In the end however the result will be the same. We start out with

Lemma. $\mathscr{K}_{S}^{\prime} \subset \mathscr{K}^{\prime}$.

Proof. Let $\lambda+\varrho \in X^{+}$and $w \in W$ such that $w \cdot \lambda \in X_{S}^{+}$. Suppose there exists $\alpha \in R^{+} \backslash R_{S}^{+}$with $\langle w(\lambda+\varrho), \check{\alpha}\rangle \geq 0$. We have to show that $\left[D_{S}(w \cdot \lambda)\right] \in \mathscr{K}^{\prime}$.

We can write

$$
\left[D_{S}(w \cdot \lambda)\right]=\sum_{y: y \cdot \lambda \in X_{S}^{+}} a_{y}\left[M_{S}(y \cdot \lambda)\right]
$$

for some $a_{y} \in \mathbb{N}$. Inserting the expression 1.2(1) in this formula we obtain

$$
\left[D_{S}(w \cdot \lambda)\right]=\sum_{y: y \cdot \lambda \in X_{S}^{+}} a_{y} \sum_{z \in W_{S}}(-1)^{l(z)}[M(z y \cdot \lambda)] .
$$

If $\lambda \notin X^{+}$then we have for each $y, z$ in (2) a reflection $s_{\beta}$ with $s_{\beta} z y \cdot \lambda=z y \cdot \lambda$, i.e. in this case $[M(z y . \lambda)] \in \mathscr{K}^{\prime}$, cf. Proposition 1.14.

If on the other hand $\lambda \in X^{+}$then there exists a simple reflection $s$ such that $w s . \lambda \in X_{S}^{+}$and $w s . \lambda<w . \lambda$. From Corollary 1.13 we get

$$
a_{y}= \begin{cases}a_{y s} & \text { if } y s . \lambda \in X_{S}^{+} \\ 0 & \text { otherwise }\end{cases}
$$


Hence we may rewrite (2) as

$$
\left[D_{S}(w \cdot \lambda)\right]=\sum_{y} a_{y} \sum_{z \in W_{S}}(-1)^{l(z)}([M(z y \cdot \lambda)]+[M(z y s \cdot \lambda)])
$$

where the first sum is now taken over those $y \in W$ for which $y . \lambda, y s . \lambda \in X_{S}^{+}$and $y . \lambda<y s . \lambda$. By Proposition 1.14 we see that $[M(z y . \lambda)]+[M(z y s . \lambda)] \in \mathscr{K}^{\prime}$ and we are done.

1.21 Proposition. $\mathscr{K}_{S}^{\prime}=\mathscr{K}^{\prime} \cap \mathscr{K}_{S}$.

Proof. By Lemma 1.20 the inclusion $\mathscr{K}_{S} \subset \mathscr{K}$ induces a homomorphism $\Phi: \mathscr{K}_{S} / \mathscr{K}_{S}^{\prime} \rightarrow \mathscr{K} / \mathscr{K}^{\prime}$. If we identify $\mathscr{K}_{S} / \mathscr{K}_{S}^{\prime}$ with $\mathscr{K}_{S}^{-}$and $\mathscr{K} / \mathscr{K}^{\prime}$ with $\mathscr{K}^{-}$ then $\Phi$ is given by

$$
\phi\left(\left[M_{S}\left(w_{S} w_{0} \cdot \lambda\right)\right]\right)=(-1)^{l\left(w_{S}\right)}\left|W_{S}\right|\left[M\left(w_{0} \cdot \lambda\right)\right], \quad \lambda \in X^{+} .
$$

To see this we just combine 1.2(1) with the fact that

$$
[M(\mu)] \equiv(-1)^{l(w)}[M(w \cdot \mu)] \bmod \mathscr{K}^{\prime}
$$

for all $\mu \in X, w \in W$, cf. Proposition 1.14.

In particular we see that $\Phi$ is injective and we are done.

1.22. Let $\mathscr{C}_{S}$ be the category of integrable $\mathfrak{p}_{S}$-modules. By restriction we may consider $\mathscr{C}$ to be a subcategory of $\mathscr{C}_{S}$. Moreover the tensor product $\otimes: \mathscr{C} \times \mathscr{C}_{S} \rightarrow \mathscr{C}_{S}$ gives the Grothendieck ring $\mathscr{R}_{S}$ of $\mathscr{C}_{S}$ (with $\mathbb{Z}$-basis $\left\{\left[L_{S}(\nu)\right] \mid \nu \in X_{S}^{+}\right\}$) an $\mathscr{B}$-algebra structure. The multiplication is given by

$$
[L(\lambda)] \cdot\left[L_{S}(\nu)\right]=\sum_{\mu \in X_{S}^{+}}\left[L(\lambda) \otimes L_{S}(\nu): L_{S}(\mu)\right]\left[L_{S}(\mu)\right], \quad \lambda \in X^{+}, \nu \in X_{S}^{+},
$$

where the multiplicity $\left[L(\lambda) \otimes L_{S}(\nu): L_{S}(\mu)\right]$ of $\left[L_{S}(\mu)\right]$ as a composition factor in $L(\lambda) \otimes L_{S}(\nu)$ is given by the classical formula

$$
\left[L(\lambda) \otimes L_{S}(\nu): L_{S}(\mu)\right]=\sum_{w \in W_{S}}(-1)^{l(w)} \operatorname{dim} L(\lambda)_{w \cdot \mu-\nu}
$$

Clearly the tensor product $\mathscr{C} \times \mathscr{\sigma}_{S} \rightarrow \mathscr{O}_{S}$ induces an $\mathscr{B}$-module structure on $\mathscr{K}_{S}$. In this way $\mathscr{K}_{S}$ is an $\mathscr{B}$-submodule of $\mathscr{K}$ and (1) gives us the formula

$$
\left[L(\lambda) \cdot\left[M_{S}(\nu)\right]=\sum_{\mu \in X_{S}^{+}}\left(\sum_{w \in W_{S}}(-1)^{l(w)} \operatorname{dim} L(\lambda)_{w, \mu-\nu}\right)\left[M_{S}(\mu)\right]\right.
$$

for all $\lambda \in X^{+}, \nu \in X_{S}^{+}$.

1.23. By Proposition 1.21 we see that $\mathscr{K}_{S}^{\prime}$ becomes an $\mathscr{B}$-submodule of $\mathscr{K}_{S}$. Moreover the proof of this proposition shows that we have an $\mathscr{B}$-module isomorphism $\Psi: \mathscr{K}_{S}^{-} \cong \mathscr{K}^{-}$given by $\Psi\left(\left[M\left(w_{S} w_{0} \cdot \lambda\right)\right]\right)=\left[M\left(w_{0} \cdot \lambda\right)\right], \lambda \in X^{+}$. Combining this with Proposition 1.18 we get

Corollary. As $\mathscr{B}$-modules we have $\mathscr{K}_{S}^{-} \cong \mathscr{B}$. The isomorphism takes $\left[M_{S}\left(w_{S} w_{0} \cdot \lambda\right)\right]$ into $[L(\lambda)], \lambda \in X^{+}$. 
Consider the full subcategory $\mathscr{Q}_{S}^{-}$of $\mathcal{Q}_{S}$ consisting of all tilting modules whose indecomposable summands have highest weights in the Weyl chamber $w_{S} w_{0} \cdot X^{+}$, i.e.

$$
M \in \mathscr{Q}_{S}^{-} \text {if and only if } M=\bigoplus_{\lambda \in X^{+}} M_{S}\left(w_{S} w_{0} \cdot \lambda\right)^{a_{\lambda}^{S}(M)}
$$

for some $a_{\lambda}^{S}(M) \in \mathbb{N}$.

Then $\mathscr{Q}_{S}^{-}$is a module for the semisimple category $\mathscr{C}$. Moreover it has a reduced tensor product given by

$$
N \underline{\otimes} M=\bigoplus_{\lambda \in X^{+}} D_{S}\left(w_{S} w_{0} \cdot \lambda\right)^{a_{\lambda}^{S}(N \otimes M)} .
$$

The corollary above says that the "fusion rules" in $\left(\mathscr{O}_{S}^{-}, \underline{\otimes}\right)$ are the same as in $(\mathscr{C}, \otimes)$.

1.24. Assume that $\mathfrak{g}$ is simple and fix $\kappa \in \mathbb{N}$. The dual Coxeter number is called $\check{h}$. Let $\tilde{\mathscr{O}}_{ \pm \kappa}$ be the category of integrable $\tilde{\mathfrak{g}}$-modules of finite length and with central charge $\pm \kappa-\check{h}$. Denote by $K\left(\tilde{\mathscr{O}}_{ \pm \kappa}\right)$ its Grothendieck ring. Let $\mathscr{J}$ be the ideal of $\mathscr{B}$ generated by the set $\left\{[L(\lambda)] \mid\left\langle\lambda+\varrho, \check{\alpha}_{0}\right\rangle=\kappa\right\}$, where $\alpha_{0}$ is the highest short root.

In [F, Theorems 4.1, 4.2] it is shown that for $\kappa \geq \check{h}+6$ then

$$
K\left(\tilde{\mathscr{O}}_{ \pm \kappa}\right) \stackrel{\sim}{\rightarrow} \mathscr{R} / \mathscr{J} \text {. }
$$

\section{Algebraic Groups}

Recall that $T \subset B \subset G$ is a maximal torus contained in a negative Borel subgroup of a semisimple, simply connected algebraic group $G$ defined over the algebraically closed field $\bar{k}$ of char $\bar{k}=p>0$. Later we shall need that $p \geq h$.

2.1. Now we let $\mathscr{C}$ denote the category of all finite dimensional representations of $G$ and if $M$ belongs to $\mathscr{C}$ then $M=\bigoplus_{\lambda \in X} M_{\lambda}$, where

$$
M_{\lambda}=\{m \in M \mid t m=\lambda(t) m, \forall t \in T\}
$$

is the $\lambda$-weight space. Note that we have identified $X$ with the character group $X(T)$ of $T$.

The induced $G$-modules will play the rôle of the dual Verma modules. We recall their definition (see e.g. [J2]). Any rational $B$-module $E$ gives rise to a rational $G$-module which we denote $H^{0}(E)$. If $E$ is finite dimensional then

$$
H^{0}(E)=\left\{f: G \rightarrow E \mid f \text { is regular and } f(g b)=b^{-1} f(g), g \in G, b \in B\right\}
$$

with $G$-action given by

$$
x f: g \mapsto f\left(x^{-1} g\right), x, g \in G, f \in H^{0}(E) .
$$

In particular if $\lambda \in X$ then $\lambda$ is a 1-dimensional representation of $B$ and we obtain the induced $G$-modules $H^{0}(\lambda)$. This module is only non-zero if $\lambda \in X^{+}$and in this case it contains a unique simple submodule which we denote $L(\lambda)$. The set $\left\{L(\lambda) \mid \lambda \in X^{+}\right\}$ is (up to isomorphisms) the full set of simple $G$-modules in $\mathscr{C}$. 
The Grothendieck group of $\mathscr{C}$ is denoted $\mathscr{B}$. It is the $\mathbb{Z}$-module with basis $\left\{[L(\lambda)] \mid \lambda \in X^{+}\right\}$. Via the tensor product in $\mathscr{C}$ we have a ring structure on $\mathscr{B}$. As in 1.15(1) we find

$$
\mathscr{B} \stackrel{\sim}{\rightarrow}[X]^{W} \text {. }
$$

Using similar notation as in the preceding section we may write

$$
\left[H^{0}(\lambda)\right]=\sum_{\mu \leq \lambda}\left[H^{0}(\lambda): L(\mu)\right][L(\mu)],
$$

and

$$
[L(\lambda)]=\sum_{\mu \leq \lambda}\left[L(\lambda): H^{0}(\mu)\right]\left[H^{0}(\mu)\right]
$$

with $\left[H^{0}(\lambda): L(\lambda)\right]=\left[L(\lambda): H^{0}(\lambda)\right]=1$.

The Lusztig conjecture [L1] says that the numbers $\left[H^{0}(\lambda): L(\mu)\right] \in \mathbb{N}$ and $\left[L(\mu): H^{0}(\lambda)\right] \in \mathbb{Z}$ are given in terms of the Kazhdan-Lusztig polynomials associated with the affine Weyl group $W_{p}$ corresponding to $G$.

2.2. If $M \in \mathscr{C}$ then the linear dual $M^{*}$ of $M$ with the contragradient action again belongs to $\mathscr{C}$. In analogy with 1.5 we shall consider a "contravariant" dual; namely for $M \in \mathscr{C}$ we set

$$
\delta M=\left(M^{*}\right)^{\sigma},
$$

where $\sigma$ is the automorphism of $G$ considered in [J2, 8.17]. This construction has the advantage of preserving weights, i.e., $\operatorname{dim}(\delta M)_{\lambda}=\operatorname{dim} M_{\lambda}$ for all $\lambda \in X$. In particular this means that

$$
\delta L(\lambda) \cong L(\lambda) \text { for all } \lambda \in X^{+} .
$$

We define the Weyl module with highest weight $\lambda \in X^{+}$by

$$
V(\lambda):=\delta H^{0}(\lambda)
$$

Note that $L(\lambda)$ is the unique simple quotient of $V(\lambda)$.

Remark. An alternative construction of $V(\lambda)$ would be to start by choosing a maximal weight vector $v^{+}$in the finite dimensional simple $\mathfrak{g}$-module with highest weight $\lambda$. Then $V(\lambda)$ may be obtained by reduction $\bmod p$ (i.e. tensoring by $\bar{k}$ ) of $\bar{U}_{\mathbb{Z}} v^{+}$, where $\bar{U}_{\mathbb{Z}}$ denotes the Kostant $\mathbb{Z}$-form of the universal enveloping algebra of $\mathfrak{g}$.

2.3. The linkage principle implies that $\mathscr{C}$ splits according to the orbits of the action of the affine Weyl group $W_{p}$. We set

$$
C=\left\{\lambda \in X^{+} \mid\langle\lambda+\varrho, \check{\alpha}\rangle<p \text { for all } \alpha \in R^{+}\right\}
$$

and

$$
\bar{C}=\left\{\lambda \in X \mid 0 \leq\langle\lambda+\varrho, \check{\alpha}\rangle \leq p \text { for all } \alpha \in R^{+}\right\} .
$$

Note that $C \neq \emptyset$ if and only if $p$ is bigger than or equal to $h$, the Coxeter number of the root datum. Identifying $W_{p}$ with the group generated by the reflections in the walls of $C$ we see that $\bar{C}$ is a fundamental domain for the action of $W_{p}$ on $X$. For $\lambda \in \bar{C}$ we define the full subcategory $\mathscr{C}(\lambda)$ of $\mathscr{C}$ by

$$
\mathscr{C}(\lambda)=\left\{M \in \mathscr{C} \mid[M: L(\mu)]=0 \text { unless } \mu \in W_{p} . \lambda\right\} .
$$


Just as in 1.2 we get translation functors

$$
T_{\lambda}^{\mu}: \mathscr{C}(\lambda) \rightarrow \mathscr{C}(\mu), \quad \lambda, \mu \in \bar{C},
$$

and the effect of these on Weyl modules are completely analogous to 1.3(2-4) replacing "Vermas" with "Weyls" and $W$ with $W_{p}$. Also the result in 1.4 carry over as long as $w \cdot \lambda \in X^{+}$, see [J2].

2.4. The analogue of Definition 1.5 is now

Definition (Donkin). A module $M \in \mathscr{C}$ is called a tilting module if $M$ and $\delta M$ both have filtrations whose successive quotients are Weyl modules.

We get immediately the same statements as (2) and (3) in 1.5. Instead of 1.5(4) we have

$$
V(\lambda) \text { is a tilting module if and only if } V(\lambda)=L(\lambda) \text {. }
$$

Remark. It is clear from the linkage principle that $V(\lambda)=L(\lambda)$ for all $\lambda \in \bar{C} \cap X^{+}$. However we have also for instance $V((p-1) \varrho)=L((p-1) \varrho)$ which is called the Steinberg module. It is possible (but rather complicated) to describe all $\lambda \in X^{+}$for which $V(\lambda)$ is simple, see [J2, II.8.21] and compare 1.8.

2.5. The Ext-vanishing result in 1.6 (and its proof) carry over to $\mathscr{C}$. So does Proposition 1.7 which in this context is due to Donkin and Ringel ([D] and [R]):

Proposition. Let $\lambda \in X^{+}$. Then there exists an indecomposable tilting module $D(\lambda) \in \mathscr{C}$ with the following properties:

(1) all weights $\mu$ of $D(\lambda)$ satisfy $\mu \leq \lambda$,

(2) $\operatorname{dim} D(\lambda)_{\lambda}=1$.

Moreover $D(\lambda)$ is unique up to isomorphism.

2.6. Although we shall not need it here let us mention the analogue of Proposition 1.8. Assume that $p>2(h-1)$.

Proposition. Let $\lambda \in \bar{C}$. Then $D(\lambda+(p-1) \varrho)=T_{(p-1) \varrho}^{\lambda+(p-1) p} V((p-1) \varrho)$ and this is the projective cover of $L\left(w_{0} \cdot \lambda+(p-1) \varrho\right)$ in the subcategory of $\mathscr{C}$ consisting of those modules whose highest weights satisfy $\langle\mu+\varrho, \alpha\rangle<2 p(h-1), \alpha \in R^{+}$.

The point is that the Steinberg module (see Remark 2.4) is a tilting module and it is projective in such a subcategory.

2.7. As in 1.11 we see that $\left\{[D(\lambda)] \mid \lambda \in X^{+}\right\}$constitute a basis for $\mathscr{R}$ and gives rise to the decomposition

$$
\mathscr{R}=\mathscr{R}^{-} \oplus \mathscr{R}^{\prime}
$$

with

$$
\begin{aligned}
\mathscr{R}^{-} & =\operatorname{span}_{\mathbb{Z}}\{[D(\lambda)] \mid \lambda \in C\}, \\
\mathscr{R}^{\prime} & =\operatorname{span}_{\mathbb{Z}}\left\{[D(\lambda)] \mid \lambda \in X^{+} \backslash C\right\} .
\end{aligned}
$$

Recall that Proposition 1.11 was modelled after the results in [A1] which deal with quantum groups and algebraic groups. Let us here only state the consequence (cf. Corollary 1.12)

(1) For $\lambda \in C, y, w \in W_{p}$ and $s \in W_{p}$ a reflection in one of the walls of $C$ such that $w s . \lambda, w . \lambda, y . \lambda \in X^{+}$and $w s . \lambda<w . \lambda$ we have

$$
[D(w \cdot \lambda): V(y \cdot \lambda)]= \begin{cases}{[D(w \cdot \lambda): V(y s \cdot \lambda)]} & \text { if } y s . \lambda \in X^{+} \\ 0 & \text { otherwise }\end{cases}
$$


2.8. For the rest of this section we assume that $R$ is irreducible. In order to define an action of $W_{p}$ on $\mathscr{R}$ we need to introduce the following elements of $\mathscr{R}$ :

$$
\chi(\lambda)=\sum_{n \geq 0}(-1)^{n}\left[H^{n}(\lambda)\right], \quad \lambda \in X
$$

where $H^{n}$ is the $n$ 'th right derived function of $H^{0}$. We recall that $\chi(\lambda)=[V(\lambda)]$ if $\lambda \in X^{+}$and that in general the elements satisfy the relations $\chi(\lambda)=(-1)^{l(w)} \chi(w \cdot \lambda)$ for any $\lambda \in X$ and $w \in W$. Let $s \in W_{p}$ be a reflection corresponding to one of the faces of $C$ then $s=s_{\alpha_{2}}=s_{i}$ for an $i \in I$ or $s=s_{0}$ is the reflection in the hyperplane $\left\{\lambda \in X:\left\langle\lambda+\varrho, \breve{\alpha}_{0}\right\rangle=p\right\}$, where $\alpha_{0}$ is the highest short root. For such an $s_{i}(i \in I \cup\{0\})$ we define for all $\lambda \in X^{+}$,

$$
[V(\lambda)] \cdot s_{i}:=\chi\left(\lambda \cdot s_{\imath}\right)
$$

Note that we use the right-action of $W_{p}$ on $X$. This makes the right-action $(*)$ welldefined as the left and right actions on any dominant $\lambda$ commute: $w \cdot(\lambda \cdot y)=w y^{-1} \cdot \lambda=$ $(w . \lambda) \cdot y$. We extend this to a $\mathbb{Z}$-linear action of $W_{p}$ on all of $\mathscr{R}$.

Proposition. $\mathscr{R}^{\prime}=\operatorname{span}_{\mathbb{Z}}\left\{f \in \mathscr{R} \mid f s=f\right.$ for some reflection $\left.s \in W_{p}\right\}$.

Proof. Let $\lambda \in X^{+} \backslash C$. If $\lambda$ is $p$-singular we have $[D(\lambda)]=\sum_{\mu} a_{\mu}[V(\mu)]$ with $a_{\mu}=0$ unless $\mu$ is $p$-singular. But any such $[V(\mu)]$ is fixed by some reflection.

So suppose $\lambda$ is $p$-regular. Then by $2.7(1)$ we may write $[D(\lambda)]=\sum_{\mu, s_{\mu}} a_{\mu}([V(\mu)]+$ $\left.\left[V\left(\mu \cdot s_{\mu}\right)\right]\right)$ for some weights $\mu$ in the $W_{p}$-orbit of $\lambda$ and reflections $s_{\mu} \in W_{p}$ such that $\mu$ and $\mu \cdot s_{\mu}$ both are dominant and lie in adjacent alcoves. Clearly $s_{\mu}$ fixes $[V(\mu)]+\left[V\left(\mu \cdot s_{\mu}\right)\right]$ and we are done.

2.9. For all $p$-regular weights $\lambda \in X$ and all $y \in W_{p}$ we have

$$
\chi(\lambda) \equiv(-1)^{l(y)} \chi(\lambda \cdot y) \bmod \mathscr{R}^{\prime} .
$$

Assume $y=s_{i}$ for an $i \in I \cup\{0\}$ and that $\lambda$ and $\lambda \cdot y$ belong to adjacent alcoves. In this case $\chi(\lambda)=-\chi(\lambda \cdot y)$ when the two alcoves in question are in different Weyl chambers. Otherwise $\chi(\lambda)+\chi(\lambda \cdot y) \in \mathscr{R}^{\prime}$ according to Proposition 2.8.

Lemma. For any $f \in \mathscr{R}, \lambda \in X^{+}$, and $y \in W_{p}$ we have

$$
[L(\lambda)] \cdot(f y) \equiv([L(\lambda)] \cdot f) y \bmod \mathscr{R}^{\prime} .
$$

Proof. It is enough to prove the lemma for $f=[V(\mu)], \mu \in X^{+}$, and $y=s_{\imath}$ with $i \in I \cup\{0\}$. We have

$$
\begin{aligned}
{[L(\lambda)] \cdot([V(\mu)] y) } & =[L(\lambda)] \cdot \chi(\mu \cdot y) \\
& =\sum_{\nu \in X} \operatorname{dim} L(\lambda)_{\nu} \chi(\mu \cdot y+\nu) \\
& \equiv-\sum_{\nu \in X} \operatorname{dim} L(\lambda)_{\nu} \chi(\mu+\nu) \bmod \mathscr{R}^{\prime}
\end{aligned}
$$


where we have used (1) and the $W$-invariance of the weights of $L(\lambda)$. Similarly we can compute

$$
\begin{aligned}
([L(\lambda)] \cdot[V(\mu)]) y & =\left(\sum_{\nu \in X} \operatorname{dim} L(\lambda)_{\nu} \chi(\mu+\nu)\right) y \\
& =\sum_{\nu \in X} \operatorname{dim} L(\lambda)_{\nu}(-1)^{l\left(w_{\nu}\right)}\left[V\left(w_{\nu} \cdot(\mu+\nu)\right)\right] y \\
& =\sum_{\nu \in X} \operatorname{dim} L(\lambda)_{\nu}(-1)^{l\left(w_{\nu}\right)} \chi\left(w_{\nu} \cdot(\mu+\nu) \cdot y\right) \\
& \equiv-\sum_{\nu \in X} \operatorname{dim} L(\lambda)_{\nu} \chi(\mu+\nu) \bmod \mathscr{R}^{\prime}
\end{aligned}
$$

and the lemma follows.

Corollary. $\mathscr{R}^{\prime}$ is an ideal of $\mathscr{R}$.

Proof. By the lemma we have for all $f$ with $f s=f$ that

$$
([L(\lambda)] \cdot f) s \equiv[L(\lambda)] \cdot f s=[L(\lambda)] \cdot f .
$$

This gives us a structure on $\mathscr{R}^{-} \cong \mathscr{B} / \mathscr{R}^{\prime}$ as a commutative ring.

2.10. The formula 1.15(2) says in this context that if $M \in \mathscr{C}$ and $\lambda \in X^{+}$, then

$$
[M] \cdot[V(\lambda)]=\sum_{\substack{\nu \in X^{+} \\ w \in W}}(-1)^{l(w)} \operatorname{dim} M_{w \cdot \nu-\lambda}[V(\nu)] .
$$

We can now write down the "fusion rules" in this setup.

Proposition. The structure constants for the multiplication on $\mathscr{B}^{-}$are given by the rule

$$
[V(\lambda)] \cdot[V(\mu)]=\sum_{\nu \in C} \sum_{w \in W_{p}}(-1)^{l(w)} \operatorname{dim} V(\lambda)_{w . \nu-\mu}[V(\nu)] .
$$

Proof. Combine (1) and Proposition 2.8.

This of course gives rise to a reduced tensor product structure and a rigid symmetric tensor category.

Remark. The fusion rule in this proposition was also proved by Georgiev and Mathieu in their recent preprint [GM2], see also their announcement in [GM1].

\section{Quantum Groups}

In this section we must work a little harder to obtain our results. This is mainly due to the choice we make of working with a general positive integer $l$. We can do so since Lusztig in [L2] has succeeded in defining the quantum Frobenius homomorphism for such l's. However he needs to work over a modification of the (by now usual) quantum algebra in which one has divided out the semisimple part and replaced it by a set of orthogonal idempotents each corresponding to a weight. It is because of the necessity of working with this modification that we shall be somewhat careful in our appeal to results proven only for the (standard) quantum algebra (especially the linkage principle). 
3.1. We start out by recapitulating some basic constructions from [L2] in order to fix notation.

First we fix once and for all an integer $l \geq 1$. We let $l^{\prime}$ be $l$ or $2 l$ if $l$ is odd and $2 l$ if $l$ is even. We set $\mathscr{A}=\mathbb{Z}\left[v, v^{-1}\right]$ and let $\mathscr{b}^{\prime}$ be the quotient ring of $\mathscr{A}$ by the ideal generated by the $l^{\prime}$-th cyclotomic polynomial. Note that $v^{2} \in \mathscr{A}^{\prime}$ has order $l$. We also set $l_{\imath}=\frac{l}{\left(l, d_{i}\right)}$ for all $i \in I$.

We shall be using two "root data." They are defined like this:

- $(I, \cdot, X, Y)$ is the quadruple where for all $i, j \in I$ we have $i \cdot j=d_{i} a_{\imath j}$. Now we identify $X$ with $\mathbb{Z}[I]$ and following Lusztig we include $I$ in $X$ by $i \mapsto i^{\prime}$. Set $Y:=\operatorname{Hom}_{\mathbb{Z}}(X, \mathbb{Z})$ and include $I$ in $Y$ by $i \mapsto i$. The pairing $\langle\cdot, \cdot\rangle: Y \times X \rightarrow \mathbb{Z}$ is defined by setting $\left\langle i, j^{\prime}\right\rangle=a_{\imath \jmath}$ for all $i, j \in I$.

- $\left(I, \circ, X^{*}, Y^{*}\right)$ is the quadruple where for all $i, j \in I$ we have $i \circ j=l_{\imath} l_{\jmath}(i \cdot j)=$ $\left(l, d_{\imath}\right)^{-1}\left(l, d_{j}\right)^{-1} l^{2} d_{i} a_{\imath \jmath}$. We set

$$
X^{*}=\left\{\zeta \in X \mid \forall i \in I:\langle i, \zeta\rangle \in l_{i} \mathbb{Z}\right\} \subset X
$$

and $Y^{*}=\operatorname{Hom}_{\mathbb{Z}}\left(X^{*}, \mathbb{Z}\right)$. We include $I$ in $X^{*}$ resp. in $Y^{*}$ by $i \mapsto i_{*}^{\prime}=l_{i} i^{\prime}$ resp. $i \mapsto i_{*}=i / l_{i}$. The pairing $\langle\cdot, \cdot\rangle: Y^{*} \times X^{*} \rightarrow \mathbb{Z}$ is then given by

$$
\left\langle i_{*}, j_{*}^{\prime}\right\rangle=\left\langle i / l_{i}, l_{j} j^{\prime}\right\rangle=\frac{l_{j}}{l_{\imath}} a_{\imath \jmath}, \quad \forall i, j \in I .
$$

With these choices there is a canonical monomorphism $\psi: X \rightarrow X^{*}$ sending $i^{\prime}$ to $i_{*}^{\prime}$.

3.2. We record the following connection between the two root data.

Lemma. Assume that the Cartan matrix $\left(a_{\imath j}\right)_{\imath, j \in I}$ is indecomposable. Then if $l$ is prime to all $a_{\imath j}$ with $i \neq j$ the matrices $\left(\left\langle i, j^{\prime}\right\rangle\right)_{i, j \in I}$ and $\left(\left\langle i_{*}, j_{*}^{\prime}\right\rangle\right)_{\imath, j \in I}$ are equal. If on the other hand $d_{\imath}$ divides $l$ for some $i \in I$ then the matrices are the transposed of each other.

Proof. Case by case analysis of the definitions.

In order to work this twist into our constructions we let - for later use ${ }^{\varepsilon} A=\left({ }^{\varepsilon} a_{\imath j}\right)_{i, \jmath \in I}$ be the Cartan matrix $\left(\left\langle i_{*}, j_{*}^{\prime}\right\rangle\right)_{i, \jmath \in I}$, where we no longer have assumed that $\left(a_{i j}\right)_{i, j \in I}$ is indecomposable. Note that if $l$ is prime to all the nonzero $a_{\imath j}$ 's then ${ }^{\varepsilon} A=\left(a_{\imath j}\right)_{\imath, \jmath \in I}$. The "pre-superscript" $\varepsilon$ should indicate that only a negligible change has been made.

3.3. Now Lusztig defines a Hopf algebra associated to each such root datum. As usual this is first done over the fraction field $\mathbb{Q}(v)$ of $\mathscr{\ell}$. Then one finds an $\mathscr{b}$-lattice in there. The quantum algebra over $\mathbb{Q}(v)$ associated to $(I, \cdot, X, Y)$ is denoted $U$ and its A-form $U \subset U$. The one associated to $\left(I, \circ, X^{*}, Y^{*}\right)$ is denoted $U^{*}$ with $\mathscr{A}$-form $U^{*}$. When dealing with a quantum algebra it is preferable to have a presentation of it by generators and relations. Although this is not a priori the case for $U$ one can in fact find such a presentation, [L2, Corollary 33.1.5]. These algebras all have canonical bases.

Tensorizing the $\mathscr{f}$-forms with a (commutative) $\mathscr{b}$-algebra $k$ we denote the resulting quantum algebras by ${ }_{k} U$ resp. ${ }_{k} U^{*}$.

3.4. We will be using the symbols $E_{\imath}^{(n)}$ and $F_{i}^{(n)}$ to denote the divided power generators in all the quantum algebras. The "semisimple" part is generated by the elements $K_{\mu}$ with $\mu \in Y$. 
As for the coefficients in the various formulas we also adopt the notation in [L2]; e.g. $v_{\imath}=v^{i \cdot i / 2}=v^{d_{i}} \in \mathscr{C}$ and $v_{\imath}^{*}=v^{i \circ i / 2}=v_{i}^{l_{i}^{2}}=v^{d_{i} \frac{l^{2}}{\left(l, d_{i}\right)^{2}}} \in \mathscr{A}$. Furthermore we set

$$
\left[\begin{array}{c}
m \\
s
\end{array}\right]_{i}=\prod_{t=1}^{s} \frac{v_{i}^{m-t+1}-v_{\imath}^{-(m-t+1)}}{v_{\imath}^{t}-v_{i}^{-t}}
$$

and

$$
\left[\begin{array}{c}
m \\
s
\end{array}\right]_{i}^{*}=\prod_{t=1}^{s} \frac{\left(v_{i}^{*}\right)^{m-t+1}-\left(v_{i}^{*}\right)-^{(m-t+1)}}{\left(v_{\imath}^{*}\right)^{t}-\left(v_{\imath}^{*}\right)^{-t}} .
$$

For any other unexplained notation we safely refer the reader to [L2].

3.5. The modification of $U$ is called $\dot{U}$. It no longer has units (and hence no semisimple part). Consider the projections

$$
\pi_{\lambda, \lambda^{\prime}}: U \rightarrow U /\left(\sum_{\mu}\left(K_{\mu}-v^{\langle\mu, \lambda\rangle}\right) U+\sum_{\mu} U\left(K_{\mu}-v^{\left\langle\mu, \lambda^{\prime}\right\rangle}\right)\right)
$$

for $\lambda, \lambda^{\prime} \in X$. Then we define

$$
\dot{U}:=\bigoplus_{\lambda, \lambda^{\prime} \in X} \pi_{\lambda, \lambda^{\prime}}(U)
$$

We see that $1_{\lambda}=\pi_{\lambda, \lambda}(1) \in \dot{U}$ satisfy $1_{\lambda} 1_{\lambda^{\prime}}=\delta_{\lambda \lambda^{\prime}} 1_{\lambda}$ hence they form a set of orthogonal idempotents in $\dot{U}$. Moreover note that $\pi_{\lambda, \lambda^{\prime}}(U)=1_{\lambda} \dot{U} 1_{\lambda^{\prime}}$. In fact it is easy to see that in $\dot{U}$ we have relations like

$$
\begin{aligned}
E_{i}^{(n)} 1_{\zeta} & =1_{\zeta+n \imath^{\prime}} E_{\imath}^{(n)}, \\
E_{i}^{(n)} F_{j}^{(m)} 1_{\zeta} & =F_{j}^{(m)} E_{\imath}^{(n)} 1_{\zeta}, \quad i \neq j,
\end{aligned}
$$

and

$$
E_{\imath}^{(n)} 1_{\lambda} F_{i}^{(m)}=\sum_{t \geq 0}\left[\begin{array}{c}
n+m+\langle i, \lambda\rangle \\
t
\end{array}\right]_{i} F_{i}^{(m-t)} 1_{\lambda+(n+m-t) \iota^{\prime}} E_{i}^{(n-t)},
$$

and one can realize $\dot{U}$ as an algebra (without 1) over a certain ring with relations like these. In $\dot{U}$ there is an $\mathscr{b}$-form that comes from the $\mathscr{b}$-form of $U$. We shall omit the details which can be found in [L2].

3.6. Let from now on $k$ be a commutative $\mathscr{A}$-algebra. The $\mathscr{A}$-subalgebra of ${ }_{\ell} U$ generated by all $K_{\mu}, \mu \in Y$ and all products

$$
\pi(i, t):=\prod_{r=1}^{t} \frac{\tilde{K}_{i} v_{\imath}^{1-r}-\tilde{K}_{-i} v_{i}^{r-1}}{v_{i}^{r}-v_{\imath}^{-r}}, \quad i \in I, t \geq 1
$$

is called $U_{\mathscr{C}} U^{0}$. Here $\tilde{K}_{ \pm i}$ is $K_{ \pm d_{i} i}$. We make every $\lambda \in X$ into a character on ${ }_{k} U^{0}$ in the standard manner: $\lambda\left(K_{\mu}\right):=v^{\langle\mu, \lambda\rangle}$ and $\lambda(\pi(i, t)):=\left[\begin{array}{c}\langle i, \lambda\rangle \\ t\end{array}\right]_{\imath}$.

Let $M$ be a ${ }_{k} U$-module. The $\lambda$-weight space of $M$ is the $k$-submodule

$$
M_{\lambda}=\left\{m \in M \mid u \cdot m=\lambda(u) m, \text { for all } u \in{ }_{k} U^{0}\right\} .
$$


The category of ${ }_{k} U$-modules which are direct sums of their weight spaces and on which the $E_{\imath}^{(n)}$,s and the $F_{i}^{(n)}$,s locally act as zero for large enough $n$ is called the category of integrable ${ }_{k} U$-modules and is denoted ${ }_{k} \mathscr{C}$.

The category of unital ${ }_{k} \dot{U}$-modules is the category of ${ }_{k} \dot{U}$-modules on which the operator $\sum_{\zeta \in X} 1_{\zeta}$ is well-defined and acts as the identity. A unital module $M$ is said to be integrable if for all $m \in M$ there is an $n \gg 0$ such that $\sum_{\zeta} E_{i}^{(n)} 1_{\zeta} m=$ $\sum_{\zeta} F_{i}^{(n)} 1_{\zeta} m=0$. The category of integrable ${ }_{k} \dot{U}$-modules is denoted ${ }_{k} \dot{\mathscr{C}}$. This tells us how to think of the $1_{\zeta}$ 's, namely as projections onto "weight spaces" and apparently $\sum_{\zeta} 1_{\zeta}$ is a "substitution" for a unit element.

We of course have similar notions attached to ${ }_{k} U^{*}$ and ${ }_{k} \dot{U}^{*}$ (so that ${ }_{k} \mathscr{C}^{*}$ and ${ }_{k} \dot{\mathscr{C}}^{*}$ are defined.)

3.7. We quote from [L2] two results. The first is only stated implicitly in [L2, 31.1.6-7, 23.1.4]; it is the fact that:

$$
\text { The categories }{ }_{k} \mathscr{C} \text { and }{ }_{k} \dot{\mathscr{C}} \text { are equivalent. }
$$

The second result is the existence of a quantum Frobenius homomorphism. Assume $k$ is also an $\mathscr{C}^{\prime}$-algebra.

Theorem [L2, 35.1.9]. There is a unique k-algebra homomorphism $\mathrm{Fr}:{ }_{k} \dot{U} \rightarrow{ }_{k} \dot{U}^{*}$ given by

$$
\begin{aligned}
& \operatorname{Fr}\left(E_{i}^{(n)} 1_{\zeta}\right)= \begin{cases}E_{i}^{\left(n / l_{i}\right)} 1_{\zeta} & \text { if } n \in l_{i} \mathbb{Z} \text { and } \zeta \in X^{*} \\
0 & \text { otherwise }\end{cases} \\
& \operatorname{Fr}\left(F_{i}^{(n)} 1_{\zeta}\right)= \begin{cases}F_{i}^{\left(n / l_{i}\right)} 1_{\zeta} & \text { if } n \in l_{i} \mathbb{Z} \text { and } \zeta \in X^{*} \\
0 & \text { otherwise }\end{cases}
\end{aligned}
$$

for all $i \in I, \zeta \in X$, and $n \in \mathbb{N}$.

3.8. From now we shall assume that $k$ is an $A^{\prime}$-algebra which also is a field of characteristic $p \geq 0$ with $\bar{k}$ as its algebraic closure.

In order to be able to use Theorem 3.7 we will relate the category ${ }_{k} \dot{\mathscr{C}}^{*}$ to the category of rational representations of the semisimple, simply connected algebraic $\bar{k}$-group ${ }^{\varepsilon} G$ associated to ${ }^{\varepsilon} A$. Moreover we take ${ }^{\varepsilon} G$ to be defined and split over $k$. Note that we may consider $k$ as a $\mathbb{Z}\left[v, v^{-1}\right]$-algebra by mapping $v$ to 1 and that this algebra homomorphism factors through the $\mathbb{Z}$-algebra structure on $k$ (by mapping $\mathbb{Z}$ onto the prime field of $k$, of course). We denote by $k_{0}$ the field $k$ considered as an $\mathscr{b}$ - or $\mathbb{Z}$-algebra in this way. We let $\overline{\mathscr{C}}^{\text {loc }}$ be the category of locally finite $\bar{U}_{k_{0}}$-modules where $\bar{U}_{k_{0}}$ is the hyperalgebra over $k$ of ${ }^{\varepsilon} G$. Recall that $\bar{U}_{\mathbb{Z}} \otimes \mathbb{Q}$ is generated by $\bar{E}_{i}, \bar{F}_{i}$ for $i \in I$ and that they together with $\bar{H}_{i}=\left[\bar{E}_{i}, \bar{F}_{\underline{\imath}}\right]$ satisfy the relations $\left[\bar{H}_{\imath}, \bar{E}_{\jmath}\right]={ }^{\varepsilon} a_{i \jmath} \bar{E}_{j},\left[\bar{H}_{i}, \bar{F}_{\jmath}\right]=-{ }^{\varepsilon} a_{i j} \bar{F}_{j},\left[\bar{H}_{i}, \bar{H}_{j}\right]=0,\left[\bar{E}_{\imath}, \bar{F}_{\jmath}\right]=\delta_{\imath \jmath} \bar{H}_{i}$, and ad $\bar{E}_{i}^{1-{ }^{\varepsilon} a_{i \jmath}}\left(\bar{E}_{j}\right)=\operatorname{ad} \bar{F}_{i}^{1-{ }^{\varepsilon} a_{i \jmath}}\left(\bar{F}_{j}\right)=0$. We shall prove

Proposition. There is an isomorphism of categories ${ }_{k} \dot{\mathscr{C}}^{*} \rightarrow \overline{\mathscr{C}}^{\text {loc }}$.

This is not a surprising result; in [L2] Lusztig refers to ${ }_{k} \dot{U}^{*}$ as the quasi-classical case (at least if $\operatorname{char}(k)$ is zero). The category $\overline{\mathscr{C}}^{\text {loc }}$ identifies with the category of 
rational ${ }^{\varepsilon} G$-modules. For a note on the origins of this result see [J2, II.1.20]. This category is slightly bigger than the category $\mathscr{C}$ considered in 2.1 as rational modules are direct limits of modules from $\mathscr{C}$. This proposition of course allows us to carry over several results from the representation theory of ${ }^{\varepsilon} G$ to ${ }_{k} \dot{\mathscr{C}}^{*}$.

Proof of proposition. Let $\mathscr{C}^{\prime} \stackrel{\phi}{\longrightarrow} k$ be the $\mathscr{b}^{\prime}$-algebra structure on $k$. First we construct a $k$-algebra isomorphism

$$
k_{0} \dot{U}^{*} \rightarrow{ }_{k} \dot{U}^{*} .
$$

Let $R$ be the $l^{\prime}$-th cyclotomic field and note that $\mathscr{l}^{\prime}$ is the ring of integers in this number field. The inclusion $\mathscr{A}^{\prime} \subseteq R$ is called $\tau$. By $R_{0}$ we denote as above the $\mathscr{A}$ algebra structure on $R$ given by $v \mapsto 1$. We can now apply [L2, Proposition 33.2.3] and obtain an isomorphism of $R$-algebras

$$
f:{ }_{R_{0}} \dot{U}^{*} \rightarrow{ }_{R} \dot{U}^{*}
$$

In loc.cit. we also find the definition of $f$ :

$$
f\left(E_{\imath} 1_{\zeta}\right)=\tau\left(v_{i}^{*}\right)^{a_{\imath}\langle i, \zeta\rangle} E_{i} 1_{\zeta} \quad \text { and } \quad f\left(F_{i} 1_{\zeta}\right)=\tau\left(v_{i}^{*}\right)^{a_{i}\langle i, \zeta\rangle+1} F_{\imath} 1_{\zeta},
$$

and we compute

$$
\begin{aligned}
& f\left(E_{i}^{(n)} 1_{\zeta}\right)=\tau\left(v_{i}^{*}\right)^{n a_{\imath}\langle\imath, \zeta\rangle+\frac{n(n-1)}{2}} E_{\imath}^{(n)} 1_{\zeta}, \\
& f\left(F_{i}^{(n)} 1_{\zeta}\right)=\tau\left(v_{\imath}^{*}\right)^{n a_{i}\langle\imath, \zeta\rangle+\frac{n(n+1)}{2}} F_{i}^{(n)} 1_{\zeta} .
\end{aligned}
$$

As $\tau\left(v_{i}^{*}\right)= \pm 1$ we see that $f$ maps the subalgebra ${ }_{{ }_{1}^{\prime}} \dot{U}^{*}$ of ${ }_{R_{0}} \dot{U}^{*}$ isomorphically onto ${ }_{A^{\prime}} \dot{U}^{*} \subseteq{ }_{R} \dot{U}^{*}$. Clearly this is an $\mathscr{A}^{\prime}$-algebra isomorphism and we obtain (*) by tensoring with $k$. Of course this gives us an isomorphism ${ }_{k} \dot{\mathscr{C}}^{*} \rightarrow{ }_{k_{0}} \dot{\mathscr{C}}^{*}$.

All we need to prove now is that there is a functor $\iota:{ }_{k_{0}} \dot{\mathscr{C}}^{*} \rightarrow \overline{\mathscr{C}}^{\text {loc }}$ which is an isomorphism. From $\left[\mathrm{L} 4,6.7\right.$ (c)] we get a surjective ring homomorphism from $\mathbb{Z}_{0} U^{*}$ to $\bar{U}_{\mathbb{Z}}$ with kernel generated by the elements $K_{i}-1$ for $i \in I \subset Y$. But as $v$ is specialized to 1 all the $K_{\mu}$ 's act as the identity on integrable ${ }_{\mathbb{Z}_{0}} U^{*}$-modules. Hence we have an induced ring homomorphism ${ }_{k_{0}} U^{*} \rightarrow \bar{U}_{k_{0}}$. As $\bar{U}_{\mathbb{Z}}$ is $\mathbb{Z}$-free this obviously induce the desired isomorphism $\iota$ when we use 3.7(1).

3.9. We can use the result of Proposition 3.8 to produce induction functors on $\dot{\mathscr{C}}^{*}$. Let $\iota_{B}$ be the analogous functor from ${ }_{k} \dot{\mathscr{C}}_{B}^{*}$ to $\overline{\mathscr{C}}_{B}^{\text {loc }}$, where $\overline{\mathscr{C}}_{B}^{\text {loc }}$ is the category of locally finite modules for the hyperalgebra of a Borel subgroup ${ }^{\varepsilon} B$ of ${ }^{\varepsilon} G$ and ${ }_{k} \dot{\mathscr{C}}_{B}^{*}$ is the category of integrable (in the obvious sense) modules for the subalgebra ${ }_{k} \dot{U}_{B}^{*}$ of ${ }_{k} \dot{U}^{*}$ generated by all $F_{i}^{(n)} 1_{\zeta}$ s.t. $\zeta \in X^{*}, i \in I, n \geq 0$.

By abuse of notation we shall drop the pre-superscript ${ }^{\varepsilon}$ from now on. Recall that we have defined induction from $B$ to $G$ in 2.1.

The functor ${ }^{*} \dot{H}_{k}^{0}=\iota^{-1} \circ H^{0} \circ \iota_{B}:{ }_{k} \dot{\mathscr{C}}_{B}^{*} \rightarrow{ }_{k} \dot{\mathscr{C}}^{*}$ is also called induction. Because of Proposition 3.8 it carries all the well-known properties of $H^{0}$. Let $N$ be the number $\left|R^{+}\right|$. Define the dual of a module in the usual way using the antipode from the Hopf algebra structure.

Theorem. We have in the obvious notation

(1) (Grothendieck Vanishing) ${ }^{*} \dot{H}_{k}^{i}(\lambda)=0$ for $i>N$ and $\lambda \in X^{*}$.

(2) (Serre Duality) ${ }^{*} \dot{H}_{k}^{i}(\lambda) \cong{ }^{*} \dot{H}_{k}^{N-i}(-\lambda-2 \varrho)^{\vee}$ for $0 \leq i \leq N$ and $\lambda \in X^{*}$. 
3.10. We now turn to our primary object, that is the quantum algebra ${ }_{k} U$. We will define several subalgebras of this. Thinking of ${ }_{k} U$ as corresponding to all of $G$, the subalgebras ${ }_{k} U$ that we define will correspond to closed subgroup schemes of $G$ and they will be indexed accordingly, i.e. the subalgebra generated by $F_{\imath}^{(n)}$ and $K_{\mu}$ (for $i \in I, n \in \mathbb{N}$ and $\mu \in Y$ ) is denoted by ${ }_{k} U_{B}$ as it corresponds to the Borel subgroup $B$. The one generated by $E_{\imath}^{(n)}, F_{i}^{(n)}$, and $K_{\mu}$ (for $i \in I, 0 \leq n<l_{i}$ and $\mu \in Y$ ) is denoted ${ }_{k} U_{G_{1} T}$, where $G_{1}$ is the first Frobenius kernel of the ordinary Frobenius homomorphism of $G$, see [L3, 8.3].

Whenever there is an inclusion of algebras, say ${ }_{k} U_{H} \subset{ }_{k} U_{P}$ for $T \subseteq H \subseteq P \subseteq G$ we have an induction functor defined as in [APW, 2.8]. It takes a ${ }_{k} U_{H}$-module $E$ to $F\left(\right.$ Hom $\left._{k} U_{H}\left({ }_{k} U_{P}, E\right)\right)$, where $F$ is the finiteness functor that picks out the sum of those weight vectors on which the $E_{i}$ 's and the $F_{\imath}$ 's in ${ }_{k} U_{P}$ act locally nilpotent. This functor is denoted by

$$
H^{0}\left({ }_{k} U_{P} /{ }_{k} U_{H},-\right):{ }_{k} \mathscr{C}_{H} \rightarrow{ }_{k} \mathscr{C}_{P}
$$

where ${ }_{k} \mathscr{C}_{H}$ and ${ }_{k} \mathscr{C}_{P}$ are the categories of integrable modules.

Using the equivalence 3.7(1) we can transport these notions to ${ }_{k} \dot{U}$. Typographically this is done by putting a dot over everything.

3.11. We will examine

$$
\overline{\mathscr{C}}^{\mathrm{loc}} \cong \dot{\mathscr{C}}^{*} \stackrel{\mathrm{Fr}^{\#}}{\longrightarrow}{ }_{k} \dot{\mathscr{C}} \cong{ }_{k} \mathscr{C} \stackrel{\text { res }^{\#}}{\longrightarrow}{ }_{k} \mathscr{C}_{G_{1}},
$$

where the functor $\mathrm{Fr}^{\#}$ is induced by the Frobenius homomorphism in the following way:

Given an integrable ${ }_{k} \dot{U}^{*}$-module $M$. Let a ${ }_{k} \dot{U}$-action on $M$ be given by letting $E_{\imath}^{(n)} 1_{\zeta}\left(\right.$ resp. $\left.F_{i}^{(n)} 1_{\zeta}\right)$ act as $\operatorname{Fr}\left(E_{i}^{(n)} 1_{\psi(\zeta)}\right) \in{ }_{k} \dot{U}^{*}\left(\right.$ resp. $\left.\operatorname{Fr}\left(F_{\imath}^{(n)} 1_{\psi(\zeta)}\right)\right)$ for all $n \geq 0$, $i \in I$, and $\zeta \in X$. Recall that $\psi$ was defined in 3.1; it is used to preserve the analogy with the action of the classical Frobenius homomorphism.

We will denote $\operatorname{Fr}^{\#}(M)$ by $M^{(1)} \in{ }_{k} \mathscr{C}$ for any module $M \in{ }_{k} \dot{\mathscr{B}}^{*}$. We call $M^{(1)}$ a twisted module. Note that by restriction $M^{(1)}$ is in ${ }_{k} \mathscr{C}_{G_{1}}$, where the action of ${ }_{k} U_{G_{1}}$ factors through the co-unit (remember that ${ }_{k} U$ is a Hopf algebra). Hence $M^{(1)}$ is a trivial module for this action.

On the other hand given such a module $V \in{ }_{k} \mathscr{C}$ which is trivial when restricted to ${ }_{k} \mathscr{C}_{G_{1}}$ then there is an $M \in{ }_{k} \dot{\mathscr{C}}^{*}$ with $V \cong M^{(1)}$.

3.12. We have now reached a point in our presentation from where the arguments closely follow the usual case treated in [PW, AW, and T]. Basically the only difference is that we have to replace the $l$ in the usual arguments by a proper $l_{i}$. So we shall omit most of the details and only state the key results pointing out as we go along where the main differences are.

First we need the classification of the simple modules in ${ }_{k} \mathscr{C}_{G_{1} B}$. Here we need the following modified "restricted" region:

$$
X_{l}=\left\{\zeta \in X \mid 0 \leq\langle i, \zeta\rangle<l_{i}, \forall i \in I\right\} .
$$

Note that $X_{l} \cong X / X^{*}$. Recall from 3.1 the canonical map $\psi: X \rightarrow X^{*}$. For any $\lambda \in X$ we have a unique decomposition

$$
\lambda=\lambda^{0}+\psi\left(\lambda^{1}\right), \quad \lambda^{0} \in X_{l}, \lambda^{1} \in X .
$$

Then we find (as in [PW, 9.3.4-5] or [AW, 1.9]) 
Theorem. For each $\lambda \in X_{l}$ the simple module $L(\lambda) \in{ }_{k} \mathscr{C}$ is also simple as an object in ${ }_{k} \mathscr{C}_{G_{1}}$. Moreover for any $\lambda \in X$ the simple module $\hat{L}(\lambda) \in{ }_{k} \mathscr{C}_{G_{1} B}$ with highest weight $\lambda$ is given by

$$
\hat{L}(\lambda)=L\left(\lambda^{0}\right) \otimes \psi\left(\lambda^{1}\right)
$$

(note that $\psi\left(\lambda^{1}\right)$ is a 1-dimensional module in $\left.{ }_{k} \mathscr{C}_{G_{1} B}.\right)$

The key argument in the proof of this theorem is to see that if $\lambda \in X_{l}$ then $H_{k}^{0}(\lambda)$ contains a unique ${ }_{k} U_{B_{1}^{+-}}$-stable line $\left(B^{+}\right.$being the Borel subgroup corresponding to the positive roots).

3.13. For any root $\alpha$ we introduce the integer $l_{\alpha}$ as follows. We choose $i \in I$ and $w \in W$ such that $\alpha=w\left(\alpha_{i}\right)$ and set $l_{\alpha}=l_{i}$. Note that $l_{\alpha}$ is independent of the choice of $i$ and $w$.

Consider the induction functor $\hat{Z}=H^{0}\left({ }_{k} U_{G_{1} B} /{ }_{k} U_{B},-\right)$. This is an exact functor which satisfies

$$
\operatorname{dim} \hat{Z}(M)=\left(\prod_{\alpha \in R^{+}} l_{\alpha}\right) \operatorname{dim} M
$$

for all finite dimensional $M \in{ }_{k} \mathscr{C}_{B}$, see [L3, 8.3]. If $\lambda \in X$ then the unique minimal weight of $\hat{Z}(\lambda)$ is $\lambda-\sum_{\alpha \in R^{+}}\left(l_{\alpha}-1\right) \alpha$ and the resulting homomorphism $\hat{Z}(\lambda)^{\vee} \rightarrow \hat{Z}\left(\sum_{\alpha \in R^{+}}\left(l_{\alpha}-1\right) \alpha-\lambda\right)$ is in fact an isomorphism, see [PW, 9.6.5].

3.14. Set $\sigma_{l}=\frac{1}{2} \sum_{\alpha \in R^{+}}\left(l_{\alpha}-1\right) \alpha$. Note that $s_{\alpha_{\imath}}\left(2 \sigma_{l}\right)=2 \sigma_{l}-2\left(l_{\imath}-1\right) \alpha_{\imath}$ because $s_{\alpha_{i}}$ stabilizes $R^{+} \backslash\left\{\alpha_{i}\right\}$ and $l_{\alpha}=l_{s_{\alpha_{i}}(\alpha)}$. This means that $\sigma_{l}$ is the unique element of $X$ such that $\left\langle i, \sigma_{l}\right\rangle=l_{i}-1$ for all $i \in I$. By 3.13 we see that

$$
\hat{Z}\left(\sigma_{l}\right)^{\vee} \cong \hat{Z}\left(\sigma_{l}\right)
$$

and the standard arguments show that therefore $\hat{Z}\left(\sigma_{l}\right)$ is simple, i.e. $\hat{Z}\left(\sigma_{l}\right)=L\left(\sigma_{l}\right)$. Moreover we get as in [AW, 2.2]

Proposition. $\hat{Z}\left(\sigma_{l}\right)=L\left(\sigma_{l}\right)$ is injective in ${ }_{k} \mathscr{C}_{G_{1} B}$.

3.15. Exactly as in $[\mathrm{AW}, 2.4]$ we deduce then

Proposition. For any $M \in \overline{\mathscr{C}}^{\text {loc }}$ we get

$$
H^{i}\left({ }_{k} U /{ }_{k} U_{G_{1} B},\left(\iota_{B}^{-1} M\right)^{(1)}\right) \cong\left(\iota^{-1} H^{i}(G / B, M)\right)^{(1)} .
$$

3.16. Combining 3.12 and 3.15 we see that, for any $\lambda \in X$, we have

$$
H^{i}\left({ }_{k} U /{ }_{k} U_{G_{1} B}, \hat{L}(\lambda)\right) \cong L\left(\lambda^{0}\right) \otimes\left(\iota^{-1} H^{i}\left(G / B, \lambda^{1}\right)\right)^{(1)} .
$$

Since $H_{k}^{i}=H^{i}\left({ }_{k} U /{ }_{k} U_{G_{1} B}, \hat{Z}(-)\right)$ we get from this the following vanishing result, cf. $[\mathrm{AW}, 2.5]$.

Theorem. $H_{k}^{\imath}=0$ for $i>N$. 
3.17. Our modification of the linkage relation goes as follows: Write $\lambda \uparrow_{\alpha} \mu$ if $\lambda, \mu \in X$ are related by $\mu=s_{\alpha} \cdot \lambda+n l_{\alpha} \alpha$ for $\alpha \in R^{+}$and $n \in \mathbb{N}$ with $n l_{\alpha}<\langle\check{\alpha}, \mu+\varrho\rangle$. We say that $\lambda$ is strongly linked to $\mu$ and write $\lambda \Uparrow \mu$ if there exists a sequence $\lambda=\lambda_{1}, \lambda_{2}, \ldots, \lambda_{r}=\mu$ with $\lambda_{s} \uparrow_{\beta_{s}} \lambda_{s+1}$ for some $\beta_{s} \in R^{+}$.

Assume for a moment that our Cartan matrix $\left(a_{i j}\right)$ is indecomposable. This definition of the linkage relation together with Lemma 3.2 show that the right way to define the affine Weyl group acting on the weights is as follows: Set $\tilde{W}_{l}=W_{l}$ if all $l_{\alpha}=l$. Otherwise let $\tilde{W}_{l}$ be the group generated by the reflections $s_{i}$ for $i \in I$ together with the reflection $s_{\breve{\beta}, 1}$ defined by $s_{\breve{\beta}, 1} \cdot \lambda:=\lambda+\left(l_{\beta}-\langle\breve{\beta}, \lambda+\varrho\rangle\right) \beta$ for all $\lambda \in X$, where $\beta \in R^{+}$is the maximal (long) root of $R$. We observe that this is in agreement with Lemma 3.2 as the coroot of the maximal root of $R$ is the highest short root of the dual root system $R^{\vee}$. In fact we see that in this case $\tilde{W}_{l}$ acts on the weights $X$ in the same way as the affine Weyl group $\check{W}_{\check{l}}$ corresponding to $R^{\vee}$ acts on $X$, where $\check{l}=\frac{1}{2}\left\langle\check{\beta}, s_{\breve{\beta}, 1} \cdot(-\check{\varrho})+\check{\varrho}\right\rangle=l+d_{\beta}\langle\check{\beta}, \check{\varrho}-\varrho\rangle$. Here $\varrho$ is half the sum of the positive coroots. In order to make sense out of this we must choose an embedding of the root system $R$ in a Euclidean vector space $E$ of dimension $|I|$ with an inner product $\langle$,$\rangle . We choose the normalization of R \subset E$ such that $\left\langle\alpha_{i}, \alpha_{\imath}\right\rangle=i \cdot i=2$ for all $i \in I$ corresponding to (simple) short roots. Then both $X$ and $Y$ are naturally embedded in $E$ and the Weyl group $\tilde{W}$ acts on $X$.

Note also that there is an obvious generalization of this to non-irreducible root systems and that the Weyl group $W$ is a subgroup of $\tilde{W}_{l}$ in all cases (with its usual dot-action on $X$ ).

Having modified the linkage relation in this manner the statement and the proof of the strong linkage principle takes the usual form, cf. [T].

Theorem. Let $\chi+\varrho$ be dominant. Assume that $L(\lambda)$ is a composition factor of $H_{k}^{j}(w \cdot \chi)$, where $w \in W$ and $j \geq 0$. Then $\lambda \Uparrow \chi$.

3.18. Clearly the induced modules $H_{k}^{0}(\lambda)$ play the same rôle as their rational $G$ module counterparts. By Serre Duality $H_{k}^{N}(\lambda)$ is a highest weight module with highest weight $w_{0} . \lambda$; it has simple head $L\left(w_{0} . \lambda\right)$ and we call $H_{k}^{N}(\lambda)$ the Weyl module and denote it $V\left(w_{0} \cdot \lambda\right)$. Note that $V(\lambda) \neq 0$ iff $\lambda \in X^{+}$. A filtration of a module is called a Weyl filtration if the successive quotients are Weyl modules. If the subquotients are induced modules (i.e. isomorphic to $H_{k}^{0}(\lambda)$ for various $\lambda$ ) the module is said to have a good filtration.

Definition (Donkin). A module in ${ }_{k} \mathscr{C}$ having both a Weyl filtration and a good filtration is called a tilting module.

This definition parallels Definitions 1.5 and 2.4 completely.

3.19. Let $\mathscr{C}$ be the full subcategory of ${ }_{k} \mathscr{C}$ consisting of finite dimensional modules and let $\mathscr{R}$ be its Grothendieck ring.

The linkage principle 3.17 implies that $\mathscr{C}$ decomposes in a direct sum indexed by the $\tilde{W}_{l}$-orbits on $X$. So we may define translation functors in the standard manner and obtain results completely analogous to $2.3,2.4(1)$, Proposition 2.5 and 2.7 replacing all occurrences of the prime $p$ with the appropriate $l_{\alpha}, W_{p}$ with $\tilde{W}_{l},(p-1) \varrho$ with $\sigma_{l}$, etc. That is the tilting modules $D(\lambda)$ exist in $\mathscr{C}$. Especially we observe that, in the case treated in 3.17 of an $l_{\alpha} \neq l$, the fundamental domain for $\tilde{W}_{l}$ is the closure of $C=\left\{\lambda \in X^{+} \mid\langle\breve{\beta}, \lambda+\varrho\rangle<l_{\beta}\right\}$. 
Like in 2.8 we can define the elements $\chi_{k}(\lambda)=\sum_{n \geq 0}(-1)^{n} H_{k}^{n}(\lambda)$ of $\mathscr{B}$ and use these to define a $\tilde{W}_{l}$-action on $\mathscr{B}$. Furthermore Proposition 2.8 and Lemma 2.9 carry over to this setup. Therefore we have

Corollary. $\mathscr{R}^{-}=\operatorname{span}_{\mathbb{Z}}\{[D(\lambda)] \mid \lambda \in C\}$ is a commutative ring with unit.

3.20. As for the fusion rules we will content ourselves with a weak analogue of Proposition 2.10 which says that

Proposition. The structure constants $a_{\lambda, \mu}^{\nu}$ of $\mathscr{R}^{-}$are given by

$$
a_{\lambda, \mu}^{\nu}=\sum_{\substack{w \in \tilde{W}_{l} \\ w . \nu \in X^{+}}}(-1)^{l(w)}[V(\lambda) \otimes V(\mu): V(w \cdot \nu)]
$$

for $\nu, \lambda, \mu \in C$.

Proof. We will show the formula

$$
a_{\nu}(M)=\sum_{\substack{w \in \tilde{W}_{l} \\ w . \nu \in X^{+}}}(-1)^{l(w)}[M: V(w \cdot \nu)],
$$

where $M=\bigoplus_{\zeta \in X^{+}} D(\zeta)^{a_{\zeta}(M)}$ is a tilting module and $\nu \in C$.

By additivity it is clearly enough to show this for $M=D(\chi)$ for some $\chi \in X^{+}$. If $\chi$ is in $C$ or if $\chi$ does not belong to the $\tilde{W}_{l}$-orbit of $\nu$, the formula immediately follows from the linkage principle 3.17 and the quantum version of Proposition 2.5. So suppose $\chi \in \tilde{W}_{l} . \nu$. By $[\mathrm{A} 1,5.6]$ which is reformulated in Proposition 1.11 we may write $D(\chi)=T_{\xi}^{\chi} D(w . \xi)$ for a semi-regular weight $\xi \in \bar{C}$ and a $w \in \tilde{W}_{l}$ such that $w . \xi$ is in the lower closure of the alcove containing $\chi$. Now we take a Weyl filtration of $D(w . \xi)$ and translate it. Using that $T_{\xi}^{\chi}$ is exact we obtain a filtration of $D(\chi)$ with subquotients of the form $T_{\xi}^{\chi} V(y . \xi)$ for certain $y \in \tilde{W}_{l}$. The short exact sequences (see 1.3(4) or [A1, 5.2])

$$
0 \rightarrow V(y \cdot \chi) \rightarrow T_{\xi}^{\chi} V(y \cdot \xi) \rightarrow V(y s \cdot \chi) \rightarrow 0
$$

for $s \in\left(\tilde{W}_{l}\right)_{\xi}$ such that $y \cdot \chi<y s . \chi$, resp.

$$
0 \rightarrow V(y s \cdot \chi) \rightarrow T_{\xi}^{\chi} V(y \cdot \xi) \rightarrow V(y \cdot \chi) \rightarrow 0
$$

for $s \in\left(\tilde{W}_{l}\right)_{\xi}$ such that $y \cdot \chi>y s . \chi$ tell use that each $T_{\xi}^{\chi} \cdot V(y . \xi)$ contribute by zero to the formula (1) which then is verified.

The proposition follows by setting $M=V(\lambda) \otimes V(\mu)$ and observing that as $\nu \in C$ then $a_{\nu}(M)=a_{\lambda, \mu}^{\nu}$. Observe that this is really nothing but a repetition of the proof of 2.7(1).

3.21. Define $\mathscr{C}^{-}$to be the full subcategory of $\mathscr{C}$ all of whose objects have composition factors with highest weights in the alcove $C$. Then Proposition 3.20 tells us how to make $\mathscr{C}^{-}$into a tensor category; namely by defining the (reduced) tensor product by

$$
V(\lambda) \underline{\otimes} V(\mu)=\bigoplus_{\nu \in C} V(\nu)^{a_{\lambda, \mu}^{\nu}} .
$$


Clearly this will define a rigid monoidal category as soon as one realizes that the duality on $\mathscr{C}^{-}$takes $V(\lambda)$ to $V\left(-w_{0} \lambda\right)$. The category $\mathscr{C}^{-}$also appears in [A1] and as remarked earlier we have relied heavily on the ideas in that paper.

Even though we have not used the main theorem in [A1] let us for the record point out that the results in this section show that this theorem holds without any restrictions on $l$ :

Theorem. Let $M \in \mathscr{C}$ be a tilting module with no connected component of the form $V(\lambda)$ with $\lambda \in C$. Then for any endomorphism $f$ of $M$ the quantum trace of $f$ vanishes. In particular the quantum dimension of $D(\lambda)$ is zero for all $\lambda \in X^{+} \backslash C$.

It is moreover well-known that $\mathscr{C}^{-}$possesses a braiding $c$ given in terms of the famous $R$-matrix. It is defined by setting $c_{V, W}=P_{V, W} \circ R$, where $P_{V, W}: V \otimes W \rightarrow$ $W \otimes V$ is the usual flip, and $R$ is viewed as an operator on $V \otimes W$. This braiding is highly non-symmetric and hence it is not "counter-productive for topology" - in the words of Turaev - as the ones encountered in 1.19 and 2.10 .

3.22. Comparing our results in this section with those in the previous one we see that when $l$ is a prime we have

(1) The quantized reduced tensor category $\mathscr{C}^{-}$is equivalent to the analogous category for the corresponding semisimple algebraic group over a field of characteristic $l$.

If we compare with Finkelberg's results from 1.24 we observe that the ideal in $\mathscr{R}$ generated by the Weyl modules with highest weights on the upper wall of $C$ (i.e. analogous to the ideal $\mathscr{J}$ in 1.24 if $l>\breve{h}+6$ ) is contained in the ideal $\mathscr{R}^{\prime}$. At least if we are working in type $A$, the two ideals coincide.

(2) Suppose that the ideals are in fact equal. Then the categories from (1) are equivalent to the analogous "fusion" categories for the corresponding affine KacMoody algebra.

\section{References}

[A1] Andersen, H.H.: Tensor products of Quantized Tilting Modules. Commun. Math. Phys. 149, 149-159 (1992)

[A2] Andersen, H.H.: A New Proof of Old Character Formulas. Contemp. Math. 88, 198-207 (1989)

[APW] Andersen, H.H., Polo, P., Wen, K.: Representations of Quantum Algebras. Invent. Math. 104, 1-59 (1991)

[AW) Andersen, H.H., Wen, K..: Representations of Quantum Algebras. The Mixed Case. J. Reine Angew. Math. 427, 35-50 (1992)

[BB] Beilinson, A., Bernstein, J.: Localisation de $\mathfrak{g}$-modules. C.R. Acad. Sci. 292, 15-18 (1981)

[BK] Brylinski, J.-L., Kashiwara, M.: Kazhdan-Lusztig conjecture and holonomic systems. Invent. Math. 64, 387-410 (1981)

[CI] Collingwood, D.H., Irving, R.: A decomposition theorem for certain self-dual modules in the category $\mathscr{O}$. Duke Math. J. 58, 89-102 (1989)

[D] Donkin, S.: On tilting modules for algebraic groups. Math. Z. 212, 39-60 (1993)

[F] Finkelberg, M.: Fusion Categories. Ph.D. thesis, Harvard 1993

[GM1] Georgiev, G., Mathieu, O.: Catégorie de fusion pour les groupes de Chevalley. C.R. Acad. Sci. 315, 659-662 (1992)

[GM2] Georgiev, G., Mathieu, O.: Fusion rings for modular representations of Chevalley groups. Preprint

[H] Humphreys, J.E.: Introduction to Lie Algebras and Representation Theory. Berlin, Heidelberg, New York: Springer 1972

[I] Irving, R.: A filtered category $\mathscr{C}_{S}$ and application. Mem. of the AMS 419 (1990)

[J1] Jantzen, J.C.: Moduln mit einem höchsten Gewicht. Lecture Notes in Mathematics 750

[J2] Jantzen, J.C.: Representations of Algebraic Groups. Academic Press 1987 
[KL1] Kazhdan, D., Lusztig, G.: Representations of Coxeter groups and Hecke algebras. Invent. Math. 53, 165-184 (1979)

[KL2] Kazhdan, D., Lusztig, G.: Tensor Structures Arising From Affine Lie Algebras I-IV, preprints (1992-1993)

[L1] Lusztig, G.: Some problems in the representation theory of finite Chevalley groups. Proc. Symp. Pure Math. 37, 313-317 (1980)

[L2] Lusztig, G.: Introduction to Quantum Groups. Birkhäuser 1993

[L3] Lusztig, G.: Quantum Groups at Roots of 1. Geom. Ded. 35, 89-114 (1993)

[L4] Lusztig, G.: Finite dimensional Hopf algebras arising from quantized enveloping algebras. J. Am. Math. Soc. 82, 257-296 (1990)

[PW] Parshall, B., Wang, J.-P.: Quantum Linear Groups. Mem. of the AMS 439 (1991)

[R] Ringel, C.M.: The category of modules with good filtrations over a quasi-hereditary algebra has almost split sequences. Math. Z. 208, 209-223 (1991)

[T] Thams, L.: Two classical results in the quantum mixed case. J. Reine Angew. Math. 436, 129-153 (1993)

Communicated by A. Connes 